Portland State University

PDXScholar

8-1-1968

\title{
The electronic instrumentation for atmospheric laser propagation studies
}

Clyde Manford Brown

Portland State University

Follow this and additional works at: https://pdxscholar.library.pdx.edu/open_access_etds Let us know how access to this document benefits you.

\section{Recommended Citation}

Brown, Clyde Manford, "The electronic instrumentation for atmospheric laser propagation studies" (1968). Dissertations and Theses. Paper 456.

https://doi.org/10.15760/etd.456

This Thesis is brought to you for free and open access. It has been accepted for inclusion in Dissertations and Theses by an authorized administrator of PDXScholar. Please contact us if we can make this document more accessible: pdxscholar@pdx.edu. 
AN ABSTRACT OF THE THESIS OF Clyde Manford Brown, Jr. for the Master of Science in Applied Science presented August 23, 1968.

Title: The Electronic Instrumentation for Atmospheric Laser Propagation Studies

APPROVED BY MEMBERS OF THE THESIS COMMTTTEE:

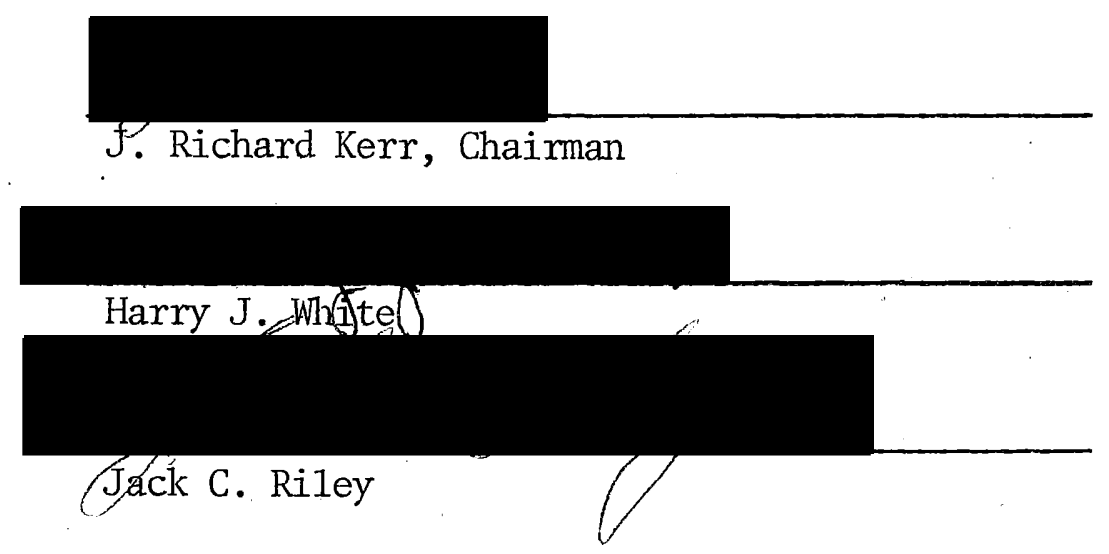

The use of laser radiation in atmospheric communication systems is revealing new forms of signal degradation not previously encountered with lower frequency transmitting sources. The coherence and directivity of the laser which is responsible for its increased signal capacity is degraded by the effect of atmospheric distortion. Random fluctuations in the index of refraction (turbulence), Rayleigh scattering, aerosol scattering, and other atmospheric effects create fluctuations (boiling, breathing, dancing, attenuation, transit-time dispersion, etc.) within the beam cross section, which when received produce random fluctuations in the receiver output signal. The two systems; which are described, are designed to investigate the effect 
of atmospheric distortions on the amplitude and phase (or transit time) of the laser beam.

The Scintillation, System was designed for real-time determination of amplitude scintillation statistics. The system will be incorporated into a multi-wavelength, multi-range experiment for comparison of theoretical and experimental meteorological and optical data. The system includes a laser transmitter, an atmospheric path over which the beam is allowed to diverge to a three-foot cross section, a pair of photomultipliers with variable spacing and small sampling apertures, an analog "scintillation computer," and a recording device. The scintillation computer is a real time, special purpose, analog computer designed to compute the variance and covariance of log amplitude, the probability distribution of $10 \mathrm{~g}$ amplitude, and the frequency spectrum of the amplitude scintillation. The system permits the recording of these data as a function of transmitter and receiver aperture, and time.

The Short Pulse Distortion System is designed to detect pulse distortion due to transit-time dispersion of the laser beam. The system includes a mode-locked laser (i.e., the optical short pulse generator), a large transmitting aperture (a Cassegrainian telescope), the atmospheric path, a wideband receiver, a sampling oscilloscope, the "Pulse Distortion Analyzer," and a high-quality tape recorder. The Pulse Distortion Analyzer is designed to compare the transmitted pulses to recorded reference pulses and to detect differential area due to time dispersion. The analyzer includes a novel, feed forward, automatic gain control circuit which utilizes a linear photo detector 
and an analog divider to smooth a $300 \mathrm{~Hz}$ signal with a 100 microsecond response time. This is necessary to smooth out the signal amplitude fluctuations due to atmospheric turbulence effects.

Both systems have been set up and tested on a one-mile path between the Oregon Graduate Center and Skyline Drive in Portland, Oregon, using a $6328 \AA$ He-Ne Laser. The ultimate use of the scintillation computer will be in a multi-wavelength experiment conducted for the Office of Naval Research, and will utilize a uniform, meteorologically instrumented path for comparison with theoretical predictions. The Pulse Distortion System will be incorporated into a joint experiment with the Electro-optic Organization of Sylvania Electronic Systems; Inc., Mountain View, California, to be conducted over a seven-mile path. 
THE ELECTRONIC INSTRUMENTATION FOR ATMOSPHERIC

LASER PROPAGATION STUDIES

by

CLYDE MANFORD BROWN, JR.

A thesis submitted in partial fulfillment of the requirements for the degree of

MASTER OF SCIENCE

in

APPLIED SCIENCE

Portland State College

1968

PORTLAND STATE COLLEGE

LIBRARY 
TO THE OFFICE OF GRADUATE STUDIES:

The members of the Committee approve the thesis of Clyde Manford Brown, Jr. presented August 23, 1968.

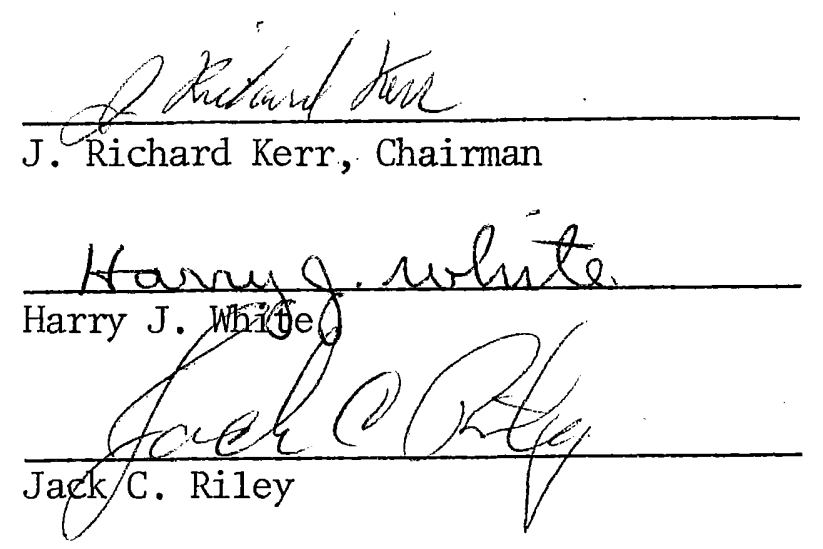

APPROVED:

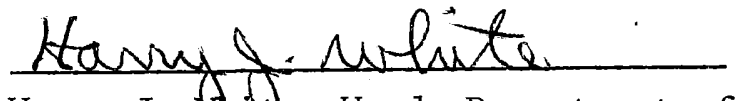

Harry J. Witte, Head, Department of Applied Science

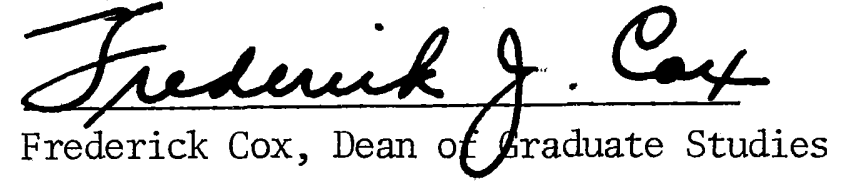


TABLE OF CONTENTS

PAGE

LIST OF FIGURES ...................... iv CHAPTER

I EXPERIMENTS ON OPTICAL PROPAGATION THROUGH

THE ATMOSPHERE ............. . . 1

General Introduction .......... 1

Amplitude Scintillations . . . . . . . 5

Transit Time Dispersion .......... . . 7

II AMPLITUDE SCINTILLATION STATISTICS . . . . . . 8

Pertinent Scintillation Statistics . . . . 8

Scintillation System . . . . . . . . 11

Receiver Configuration

Scintillation Computer

Logic Flow

Component Description

Oven and Power Supplies

Bandwidth, Dynamic Range and Calibration

Representative Results . . . . . . . 37

III PULSE DISTORTION SYSTEM . . . . . . . . . . 45

General System . . . . . . . . . 45

Distortion Analyzer Design . . . . . . . 49

Receiver Operation . . . . . . . . 57

BIBLIOGRAPHY . . . . . . . . . . . . . . . 63 


\section{LIST OF FIGURES}

FIGURE

PAGE

1. Photographs of Scintillation System . . . . . . . 3

2. Photographs of Pulse Distortion System . . . . . 4

3. Block Diagram of Scintillation System . . . . . . 12

4. Isometric Drawing of Duck-Bil1 . . . . . . . 13

5. Illustration of Large Aperture Using a Two-Foot Aluminum Mirror ............. 15

6. Block Diagram of Scintillation Computer . . . . . 17

7. Logic Flow for Computation of Variance . . . . . . 18

8. Logic Flow for Computation of Covariance . . . . . . 19

9. Logic Flow for Probability Distribution Analysis and Spectrum of Scintillations . . . . . . . . 21

10. C101, Input Amplifier and Reference Sources . . . . 22

11. C102, Logarithmic Amplifier............. 23

12. C103, High Pass Amplifier . . . . . . . . 25

13. C104, Multiplier . . . . . . . . . . . 26

14. C105, Averagex .. . . . . . . . . 27

15. C106, Channel One Averager . . . . . . . 29

16. C107, Subtractor - Differential Averager . . . . 30

17. Isometric Drawing of Oven . . . . . . . . . 31

18. Thermal Regulation Circuitry . . . . . . . . . 33

19. Intermal 15 Volt Regulator with Over-Voltage Circuit 34 
20. Curve - Covariance versus Separation with a "Tail" •

21. Curve - Covariance versus Separation . . . . . . 40

22. Curve - Variance versus Time . . . . . . . . 41

23A, Curve - Probability Distribution . . . . . . . 43

23B. Curve - Probability Distribution with Averaging . . . 43

24. Curve - Photograph of Spectrum of Amplitude Scintillations............ . . 44

25. Block Diagram of Short Pulse Distortion System . . . 46

26. Diagrams of Laser Mode Oscillation . . . . . . . 47

27. Automatic Gain Control - Divider with Transistor

Switch . . . . . . . . . . . 50

28. Block Diagram of Short Pulse System Comparator . . . 52

29. Short Pulse System Subtractor . . . . . . . . 53

30. Short Pulse System Fullwave Rectifier . . . . . . . 54

31. Short Pulse System Resetable Integrator . . . . . . 55

32. Short Pulse System Threshold Alarm . . . . . . . 56

33. Short Pulse System Logic Waveforms . . . . . . . . 59 


\section{CHAPTER I}

\section{EXPERTMENTS ON OPTICAL PROPAGATION THROUGH THE ATMOSPHERE}

\section{GENERAL INTRODUCTION}

The use of laser radiation in atmospheric communication systems is revealing new forms of signal degradation not directly encountered with lower-frequency sources. The coherence and directivity of the laser which is responsible for its increase in signal capacity is degraded by the effect of atmospheric turbulence. The turbulence creates a number of beam distortions at the receiver which have been described qualitatively as "breathing" (fluctuations in the cross-sectional area); "boiling" (a fluctuation of the bright and dark interference patterns within the beam cross section); "tearing" (gross boiling or changes in steering affecting only part of the beam width); "image dancing" (distortion of the wavefront or transverse phase); and "steering" (translation of the cross-sectional midpoint). The result of these combined distortions is that the beam cross section as intercepted by a receiver is constantly in random motion and fluctuation. This naturally results in random fluctuations in the output of the receiver which interfere with and degrade the information received. In addition, aerosol and molecular scattering cause attenuation and dispersion of the optical transit time.

The purpose of this report is to discuss two systems which have been designed and constructed to experimentally investigate the effects 
of turbulence on atmospheric propagation of the laser beam. The two systems, the Amplitude Scintillation System (Figure1) and the Short Pulse Distortion System (Figure 2), were built in conjunction with the Atmospheric Propagation Studies being conducted by Professor J. Richard Kerr at the Oregon Graduate Center. The Scintillation System is designed for future incorporation into a multi-wave length, multirange experiment for comparison of experimental data with theoretical predictions. Meteorological data will also be recorded simultaneously for comparison to optical results. The development of this system has been instrumental in the award of a research contract by the Office of Naval Research for the further support of these studies. The Short Pulse Distortion System was designed to be used in joint experiments with the Electro-Optic Organization of Sylvania Electronic Systems, Western Division, of Mountain View, California, and will be operated on their seven-mile link in the near future.

Both experiments have been put into operation on our one-mile atmospheric path between a transmitting shack located on the roof of our Iaser laboratory at the Oregon Graduate Center and a mobile receiver (Figure 1) parked near Skyline Drive. The laser used in these preliminary experiments was a Spectra Physics, Inc. Model 125 Helium-Neon Laser operating at $6328 \mathrm{~A}$ with an output power of 50 milliwatts (Figure 1). Since quantitative results require accurate knowledge of turbulence over the path, the final work will be completed on other paths, i.e., paths which are horizontal (isobaric) and uniform and which can be monitored continuously for meteorological parameters. 


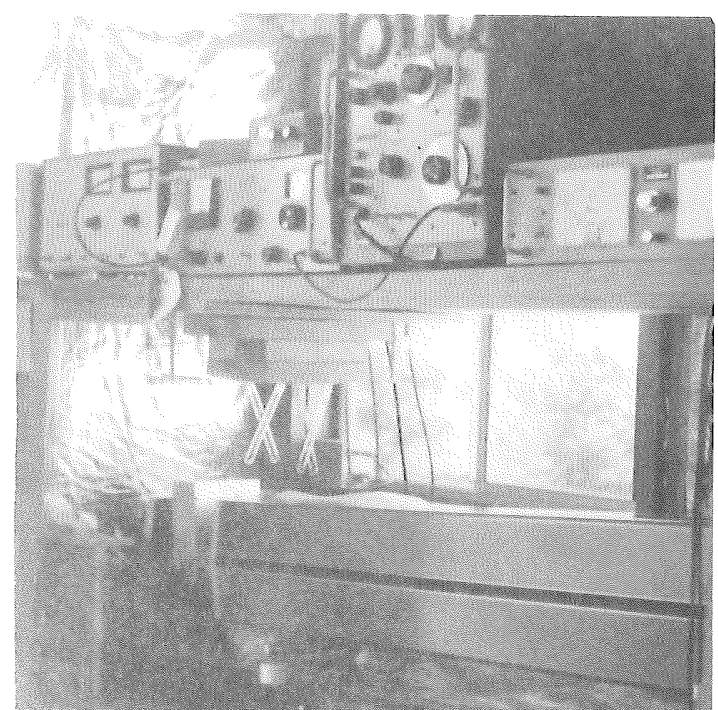

The interior of the transmitting shack showing the laser, supplies, and modulation drivers

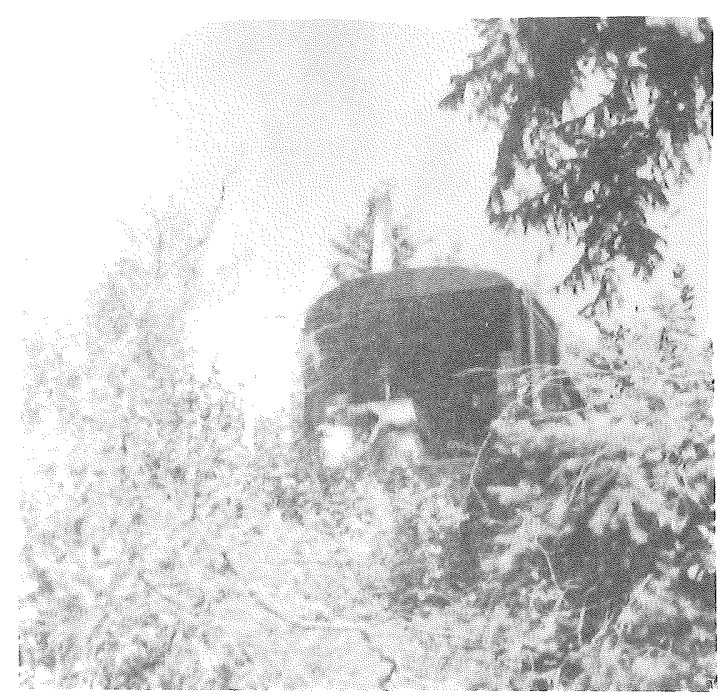

Receiver Van

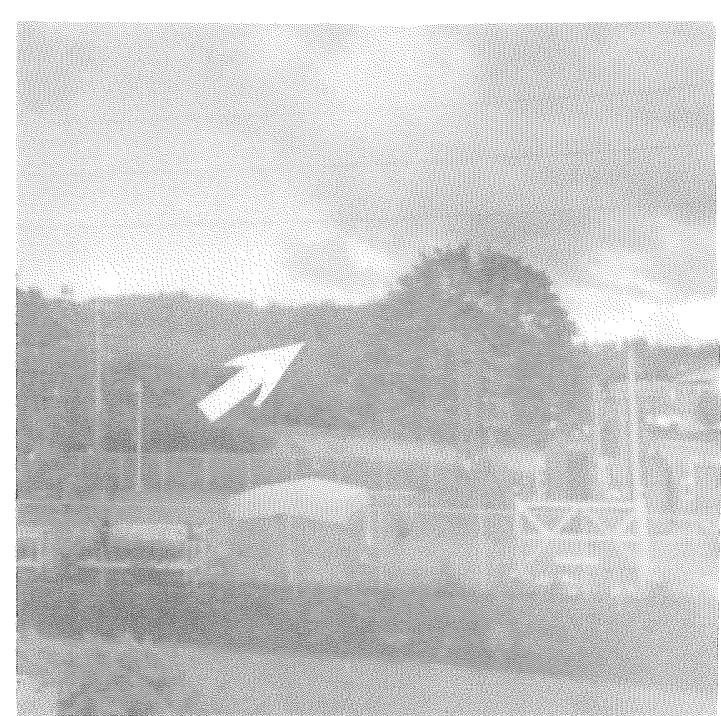

Atmospheric path between the OGC and Skyline Drive showing the general location of the receiver van

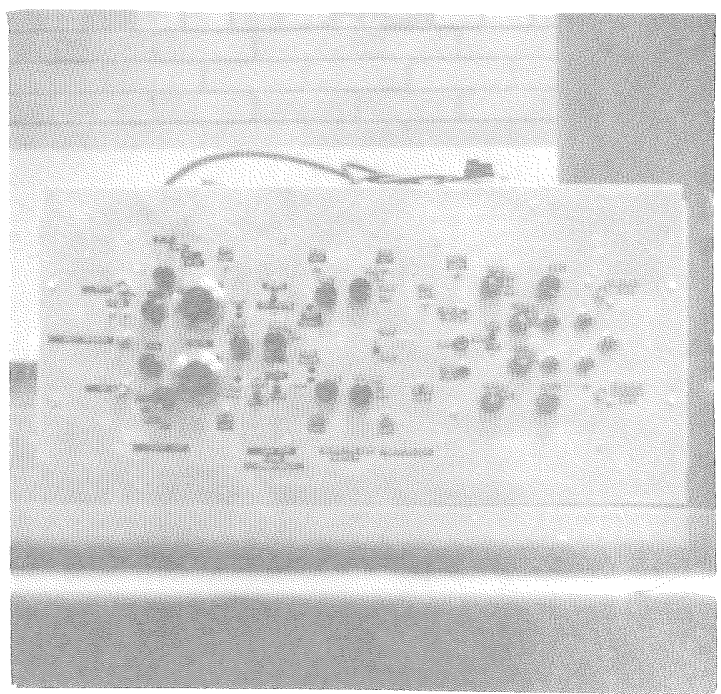

Scintillation Computer

Figure 1. Photographs of Scintillation System 


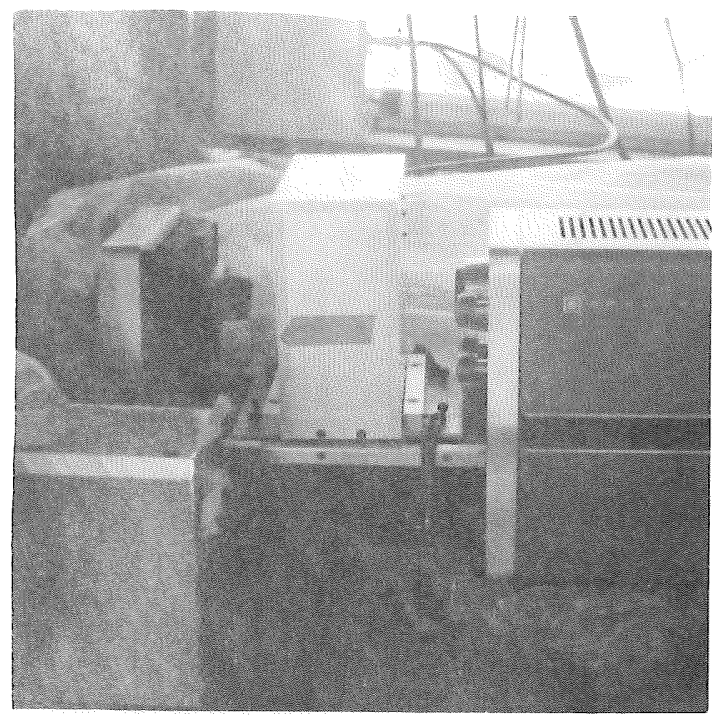

Cavity extension with in-cavity modulator

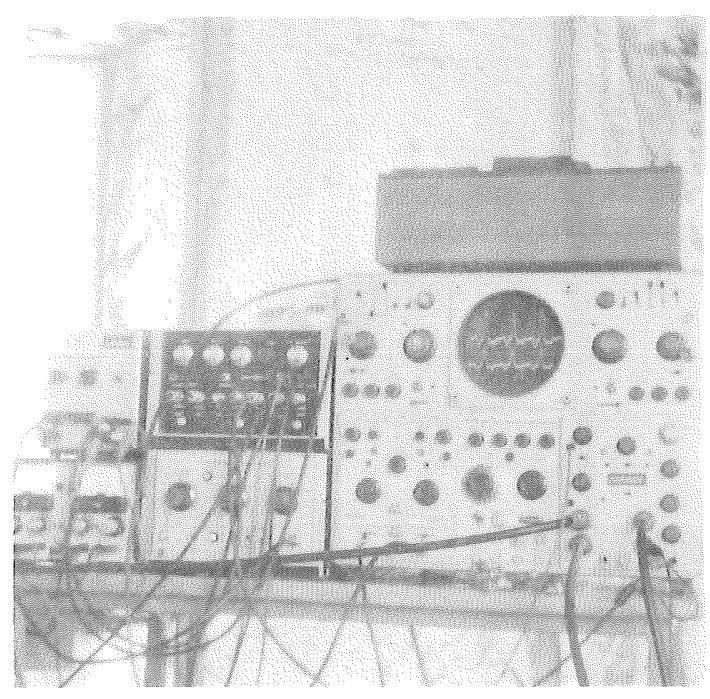

Complete Short Pulse System Receiver configuration in operation showing pulse shape on CRT

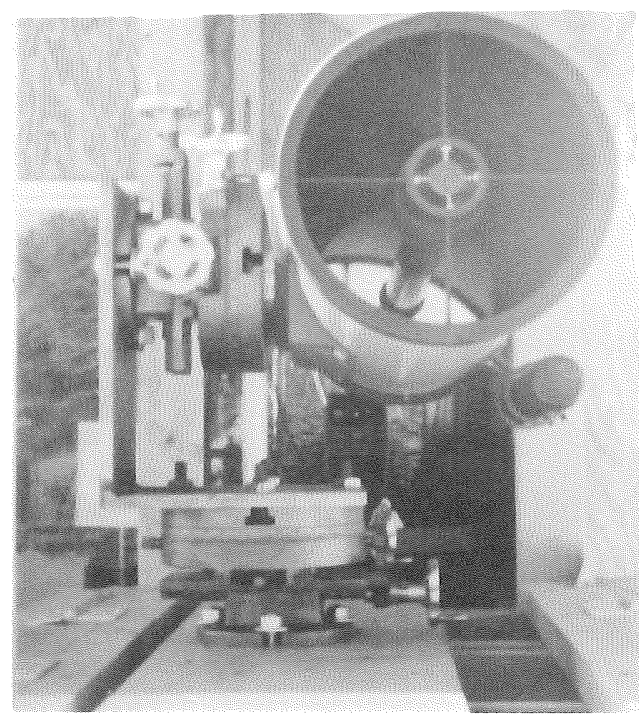

Cassegranian Telescope

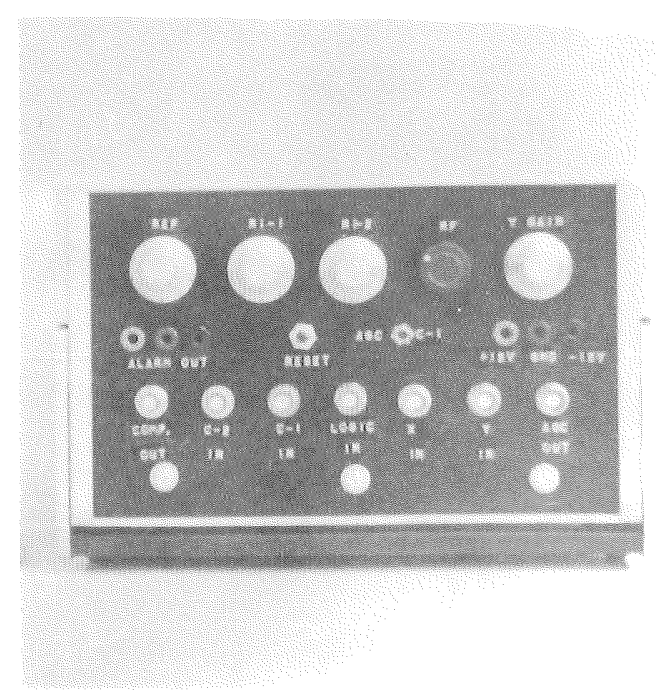

Short Pulse System Comparator

Figure 2. Photographs of Pulse Distortion System 
The Amplitude Scintillation System is designed for real-time computation of amplitude scintillation statistics at the receiver. These statistics are calculated by a real-time analog computer with various aperture sizes and spacings. The laser beam is allowed to diverge to a beam diameter of three feet at the receiver, and is received by two photomultipliers with variable spacings. The outputs of the detectors are processed by the computer to yield pertinent statistical data as described in Chapter II.

The Short Pulse Distortion System is designed to measure the propagation time dispersion of ultra-short optical pulses. The transit time dispersion or pulse smearing is highly important in determining bandwidth limitations of high-data-rate optical systems, and constitutes a convenient measure of absolute optical phase variations. Large optics are used to collimate and capture the entire laser beam, which is then focused on a fast photoreceiver. The distortion analyzer is designed to analyze the pulses "live" at the receiver and/or record them for later processing, as described in Chapter III.

Both systems comprise novel applications of state-of-the-art electronics modules to a new field of investigation. The response, accuracy, and dynamic range necessary for good results has been achieved, and it is expected that the utilization of the systems in a long-term experimental program will be successful.

\section{AMPLITUDE SCINTILLATIONS}

The primary cause of amplitude scintillations in clear air (i.e., no aerosols or contaminants) is random fluctuations of the refractive 
index of the air. Although the variations are less than one part in $10^{6}$ (1), their effects are cumulative over the path. Thus, although the mean instantaneous angular deflection due to one turbulence point is only one milliradian (1), it would result in a displacement of 5.28 feet at a receiver located one mile away. If the radiation is timecoherent as in the case of a laser, interference effects are prominent. Under isobaric conditions, the refractive index changes are related to temperature differentials by the ideal gas law (1):

$$
\frac{\Delta V}{V}=\frac{\Delta T}{T}
$$

Thus, the degree of variation of the index of refractions can be directly related to rapid temperature fluctuations, which may be measured. The pertinent relationship is (1)

$$
\Delta \mathrm{n}=\frac{-(\mathrm{n}-1)}{\mathrm{T}} \Delta \mathrm{T}
$$

The refractive index variance as determined from temperature measurements is a necessary parameter for comparison of optical scintillation results with theory.

The theoretical treatments of wave propagation in a randomly inhomogeneous medium have utilized both geometrical $(1,2)$ and physical $(3,4)$ optics. The latter approach is necessary for distances beyond a few meters, i.e., at ranges where interference between parts of the perturbed wavefront is evident. The physical-optical analyses have mainly utilized a perturbation approach and have a range of validity whose limits have not been well established $(5,6)$. Questions of ergodicity 
and stationarity also arise (7). A discussion of these theoretical issues is beyond the scope of this report; it is simply pointed out that the results of the analyses are expressions for the statistical quantities which are discussed in Chapter II, as a function of wavelength, range, degree of turbulence (refractive index variance), aperture sizes, etc.

The motivation for the experimental study of these phenomena arises from two factors. First, propagation in random media is not well understood and comprises an interesting fundamental field of investigation. Second, the statistics of the scintillating beam must be understood in order to facilitate the design of optical communications systems which will function well in such a channel (7).

\section{TRANSIT TIME DISPERSION}

The presence of aerosols in the propagation path is predicted to give rise to substantial path-length or transit-time dispersion $(8,9)$. This effect has not yet been measured, and constitutes an important limitation on the bandwidth of straight-forward optical communications systems operating in the atmosphere (7). The analyses of multipath scattering will not be discussed here, except to point out that timedispersions of up to one microsecond have been predicted under practical conditions $(8,9)$. Although there are various other possible mechanisms for such dispersion in the atmosphere (10), it appears that aerosols will predominate at currently-achievable bandwidths. The system described in Chapter III will detect dispersions on the order of $10^{-10}$ seconds lasting for one millisecond or more. 
CHAPTER II

AMPLITUDE SCINTILLATION MEASUREMENTS

The description of the Scintillation System is divided into two parts; first, a discussion of the pertinent statistics, and second, a description of the system itself.

\section{PERTINENT SCINTILLATION STATISTICS}

The propagation of laser radiation through the atmosphere gives rise to an instantaneous electromagnetic field at the receiver, which is described as a function of position $(\vec{r})$ and time $(t)$ by the complex amplitude $\tilde{\mathrm{A}}$ :

$$
\tilde{A}=\bar{A} e^{\ell(\vec{r}, t)+i \Phi(\vec{r}, t)},
$$

where $\bar{A}$ is the mean amplitude, $\ell$ is the logarithm of the instantaneous, normalized amplitude, and $\Phi$ is the phase of the wave. It is written in this form because $\ell$ is the pertinent theoretical quantity:

$$
\dot{\ell}=\log (\mathrm{A} / \overline{\mathrm{A}})=\log \mathrm{A}-\log \overline{\mathrm{A}},
$$

where $A$ is the amplitude of the light and equals the square root of the intensity.

There is considerable theoretical evidence $(3)$ that both $\ell(\vec{r}, t)$ and $\Phi(\vec{r}, t)$ are in most cases gaussian random variables, which means that $\log A$ is gaussian. Thus $A$ is said to have a "log normal 
distrubution." This is a basic concept in atmospheric propagation theory and should be investigated experimentally in detail.

There are four properties of $\ell$ which are of basic importance. The first is the probability distribution of $\ell$. Although in most cases $\&$ should have a normal (gaussian) distribution, this is not necessarily true near the edge of a confined (laser) beam (11). Since the optimum design of an optical communications system in an atmospheric channel is heavily influenced by the probability distribution of $\ell$, this anomaly is of great interest.

The second property is the covariance of $\ell$ as a function of separation in the transverse mode. Generally, the beam cross-section is isotropic ( 3 ); therefore, the separation $r$ is a scalar quantity. The covariance of $\&$ relative to points 1 and 2 is defined as (12)

$$
C_{\ell}(r)=\left(\ell_{1}-\bar{\ell}_{1}\right)\left(\ell_{2}-\bar{l}_{2}\right)
$$

This expression can be reduced in order to simplify the computation electronics. From Equation 4 we have:

$$
\ell=\log \mathrm{A}-\log \overline{\mathrm{A}}
$$

and $\bar{\ell}=\overline{\log A}-\overline{\log \bar{A}}$

$$
\overline{\log \mathrm{A}}-\log \overline{\mathrm{A}} \text {. }
$$

Therefore subtracting $\bar{l}_{1}$ from $\ell_{1}$ we obtain

$$
\begin{aligned}
\ell_{1}-\bar{\ell}_{1} & =\log A_{1}-\log \bar{A}_{1}-\overline{\log A}+\log \bar{A} \\
& =\log A_{1}-\overline{\log A} .
\end{aligned}
$$


Substituting Equation 7 into Equation 5 yields

$$
C_{\ell}(r)=\overline{\left(\log A_{1}-\overline{\log A_{1}}\right)\left(\log A_{2}-\overline{\log A_{2}}\right)} .
$$

This simplifies the computation of the covariance since the normalized amplitude drops out of the covariance calculation and need not be found explicitly. Since the covariance is a direct measure of the spacial correlation of amplitude scintillations in the transverse plane, it is highly important in the diversity reception of a scintillating laser beam ( 7 ).

The third property is the variance $C_{\ell}(0)$, which is a degenerate case of the covariance $(r=0)$. This is important since it is the quantitative measure of the degree of scintillation.

The fourth basic property of $\ell$ is its mean value $\bar{l}$. This is an important quantity and some erroneous theoretical calculations have been published (13). In the case of plane wave illumination of the atmospheric channe1, it has been theoretically shown that (3)

$$
\bar{\ell}=C_{\ell}(0) .
$$

However, for the laser case (14),

$$
\bar{\ell}=f\left(C_{\ell}(0), \lambda, r_{0}, L\right),
$$

where $\lambda$ is the wave length, $r_{0}$ is the transmitter diameter, and $L$ is the range. It is of interest to investigate this quantity experimentally in order to examine the applicability of the plane wave approximation. The log amplitude $\ell$ is defined for a point in space. However, it is also of interest to measure the probability distribution and variance 
of a signal resulting from a larger receiver aperture. When the aperture is appreciably larger than one amplitude correlation area, "aperture averaging" statistics apply (15).

Finally, the non-stationarity of the scintillation statistics under conditions of changing turbulence are of interest. Thus the variance and covariance may be considered to be a slow function of time.

\section{SCINTILLATION SYSTEM}

The Scintillation System consists of a laser, a one-mile atmospheric path, a variable-separation dual receiver, and the scintillation computer (Figure 3). The laser is a Spectra Physics Model 125, which is a $50 \mathrm{mw}, \mathrm{He}-\mathrm{Ne}$ laser operating at $6328 \AA$. The laser is situated in a shack on the roof of the laser laboratory at the Oregon Graduate Center. It is pointed at a receiver station which is a converted school bus parked in Skyline Hills Cemetery one mile away. The inherent divergence of the laser output results in a three-foot beam diameter at the receiver, and thus we did not need to use expanding optics at the transmitter. The light is received by a "duck-bill" device which provides angular selectivity while allowing investigation of the beam cross section. The beam entering the duck-bill strikes a pair of mirrors mounted on a mechanical drive which provides variable spacing in the transverse plane (Figure 4), and is then reflected into a pair of fixed photomultipliers. The front-surface, optically flat mirrors are apertured to create small sampling areas and are driven apart or together by a variable speed motor, while their spacing is read out 


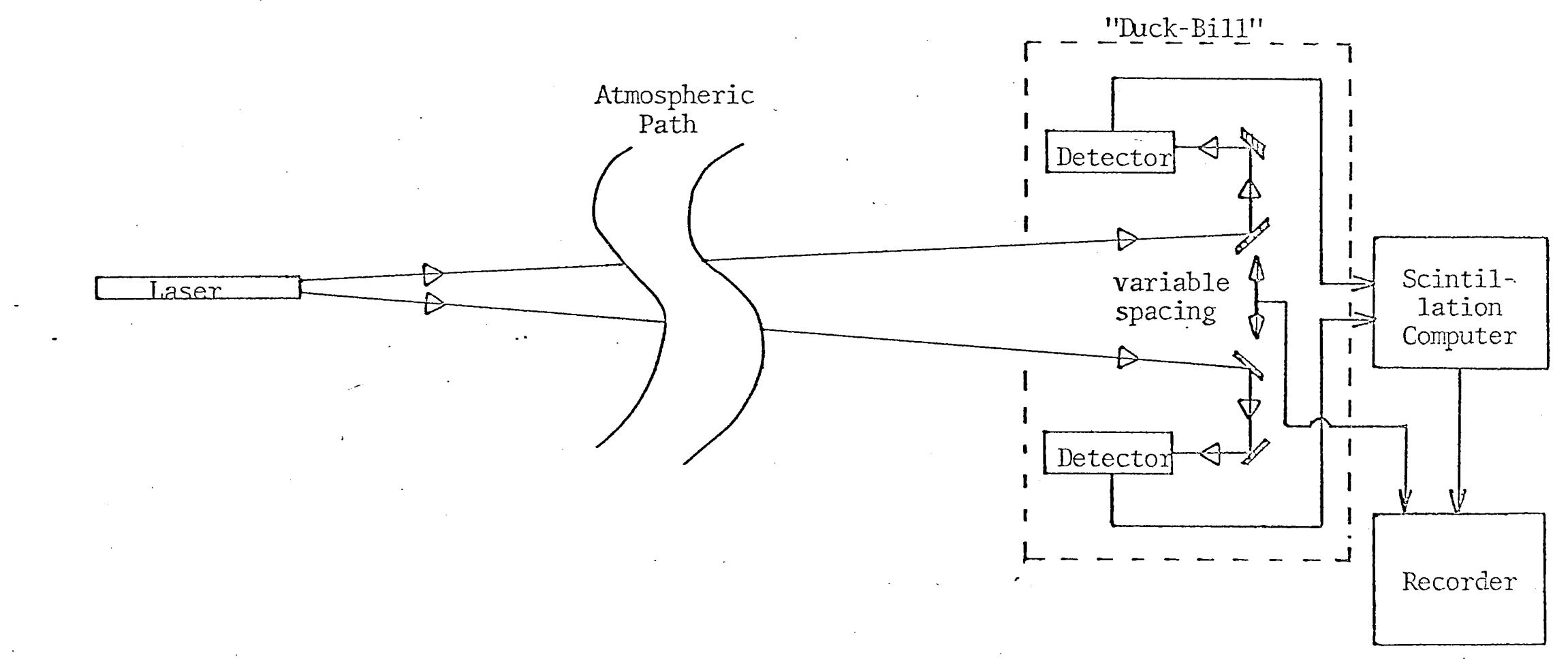

Figure 3. Block diagram of Scintillation System 


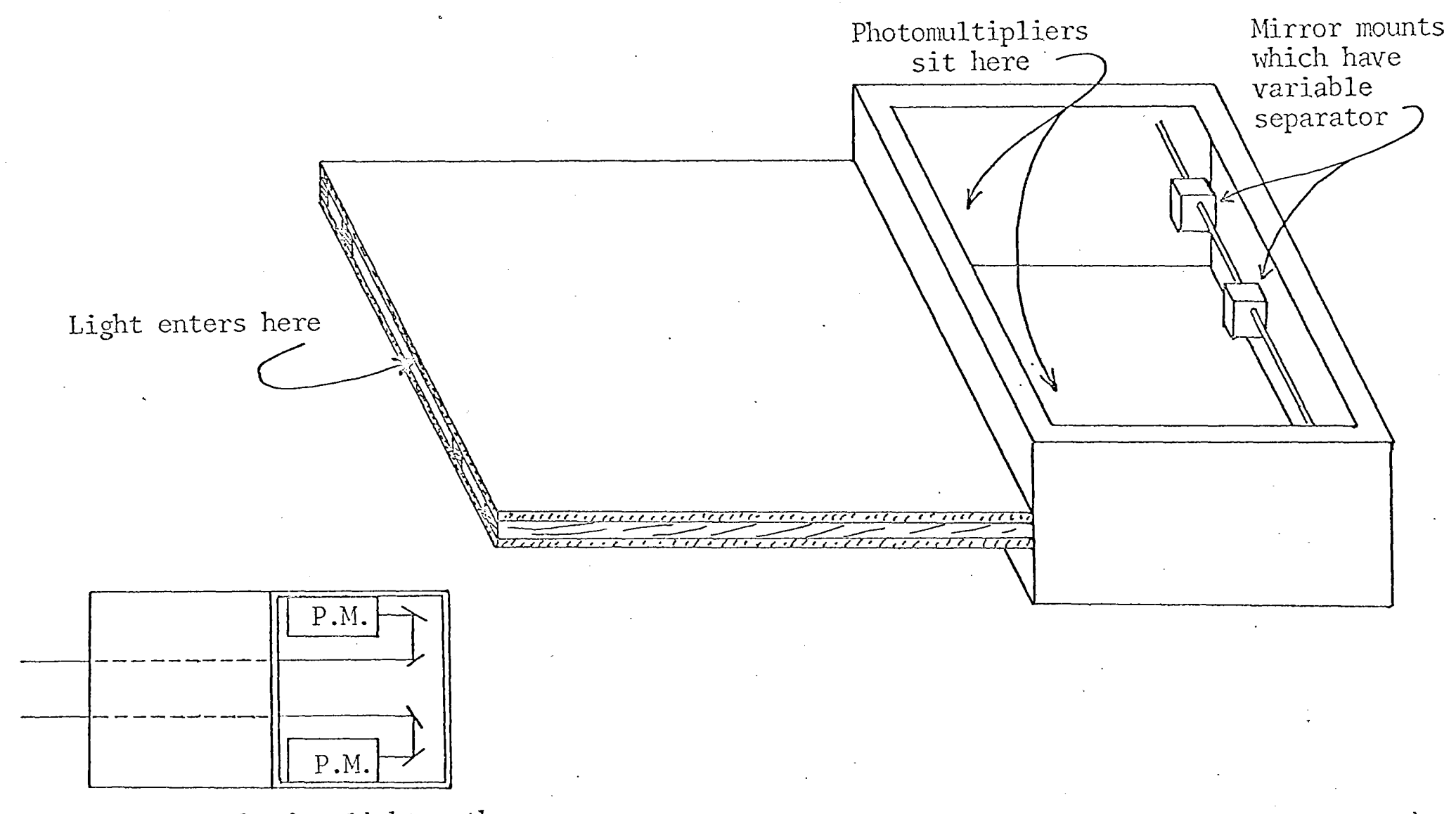

Top view showing light path

Figure 4. Isometric drawing of Duck-Bill 
by a potentiometer which is attached to the drive mechanism. The outputs from the two photomultipliers are fed into Channels No. 1 and No. 2 of the scintillation computer.

$\underline{\text { Receiver Configuration }}$

There are five relevant, real-time measurements of the pertinent amplitude statistics which are made for various turbulence conditions. The first two, the covariance of the log amplitude versus separation $(r)$ and the variance and covariance of $\log$ amplitude versus time are made utilizing the duck-bill receiver. The covariance and variance are recorded versus time utilizing a strip chart recorder and the covariance versus separation is recorded on an $X Y$ recorder with the potentiometer separation-signal driving the $y$ axis. In both measurements, the input mirrors are masked to create small sampling apertures. The remaining three measurements are the variance of $10 \mathrm{~g}$ amplitude versus receiver aperture, the probability distribution of $10 \mathrm{~g}$ amplitude versus aperture, and the spectrum of scintillations versus aperture. A two-foot diameter, 48-inch focal length, non-diffractionlimited aluminum mirror is used to provide a large maximum aperture which can be masked to create a variable pickup area. The energy reflected off this mirror is directed onto a photomultiplier as shown in Figure 5.

In the future, the computer will be replicated twice and the measurements will be conducted simultaneously at three wavelengths. Also, this will introduce the possibility of scintillation crosscorrelation measurements at two different wavelengths. 


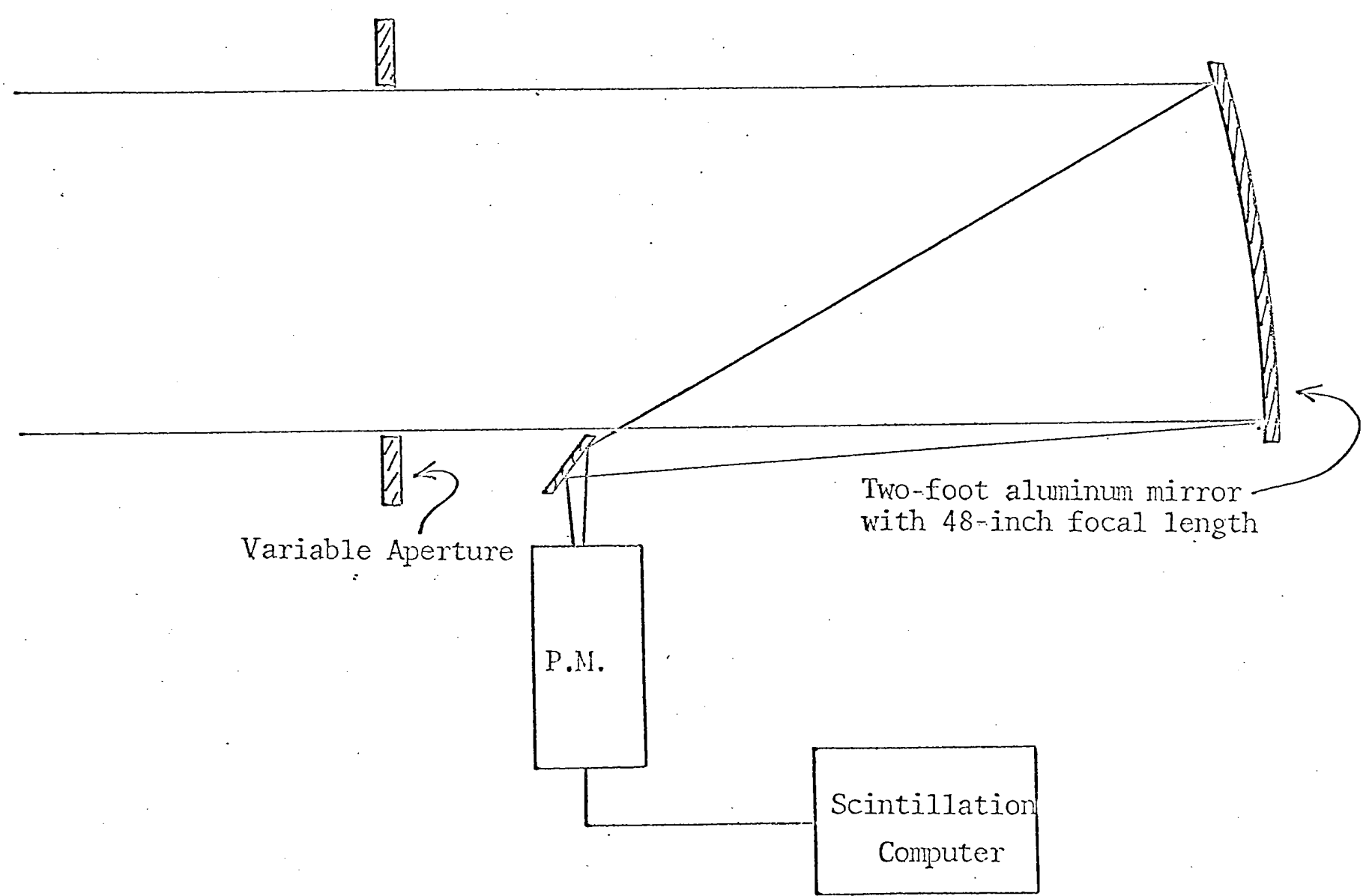

Figure 5. Illustration of large aperture using a two-foot aluminumirror 


\section{Scintillation Computer}

The scintillation computer is a real-time analog computer which has been designed to compute log variance, log covariance, log probability density and log average as previously described. It is housed in an oven-type shielded enclosure with all amplifiers balanced to eliminate drift, offset and pickup and accepts inputs from two photomultipliers (or any signals in a -.001 volt to -5 volt range). The computed results are obtained from two BNC output connectors and in addition the result of each step in the computation process is connected to a front panel connector for "live" monitoring of the individual amplifiers during operation. State-of-the-art analog function modules are used for logarithmic and multiplicative operations.

\section{Logic Flow}

For computation of variance (Figure 7 ), the photomultiplier input to Channel No. 1 is amplified by $\mathrm{C} 101$, converted to logarithm by $\mathrm{C} 102$, ac coupled by $\mathrm{C} 103$, squared by $\mathrm{C} 104$, and averaged by $\mathrm{C} 105$. The corresponding signal operations are: $\mathrm{A}, \log _{10} \mathrm{~A}, \log _{10} \mathrm{~A}-\overline{\log _{10} \mathrm{~A}}$, $\left(\log _{10} \mathrm{~A}-\overline{\log _{10} \mathrm{~A}}\right)^{2}$, and $\overline{\left(\log _{10} \mathrm{~A}-\overline{\log _{10} \mathrm{~A}}\right)^{2}}$ which has been shown to be the desired, normalized log variance of A (Equation 8 ). For computation of covariance (Figure 8 ), each channel computes $\left(\log _{10} \mathrm{~A}_{1,2}-\overline{\log _{10} \mathrm{~A}_{1,2}}\right.$ and C104 multiplies these results together. The product is then averaged by $\mathrm{C} 105$, yielding $\overline{\left(\log _{10} \mathrm{~A}_{1}-\overline{\log _{10} \mathrm{~A}_{1}}\right)\left(\log _{10} \mathrm{~A}_{2}-\overline{\log _{10} \mathrm{~A}_{2}}\right)}$ which has been shown to be the desired covariance (Equation 8 ).

In order to obtain $\ell$ or $\bar{l}$, the unit C107 operates on $\log _{10} \mathrm{~A}_{1}-\log _{10} \overline{\mathrm{A}}_{1}$ as a difference amplifier or integrator respectively. 
Scintillation Computer

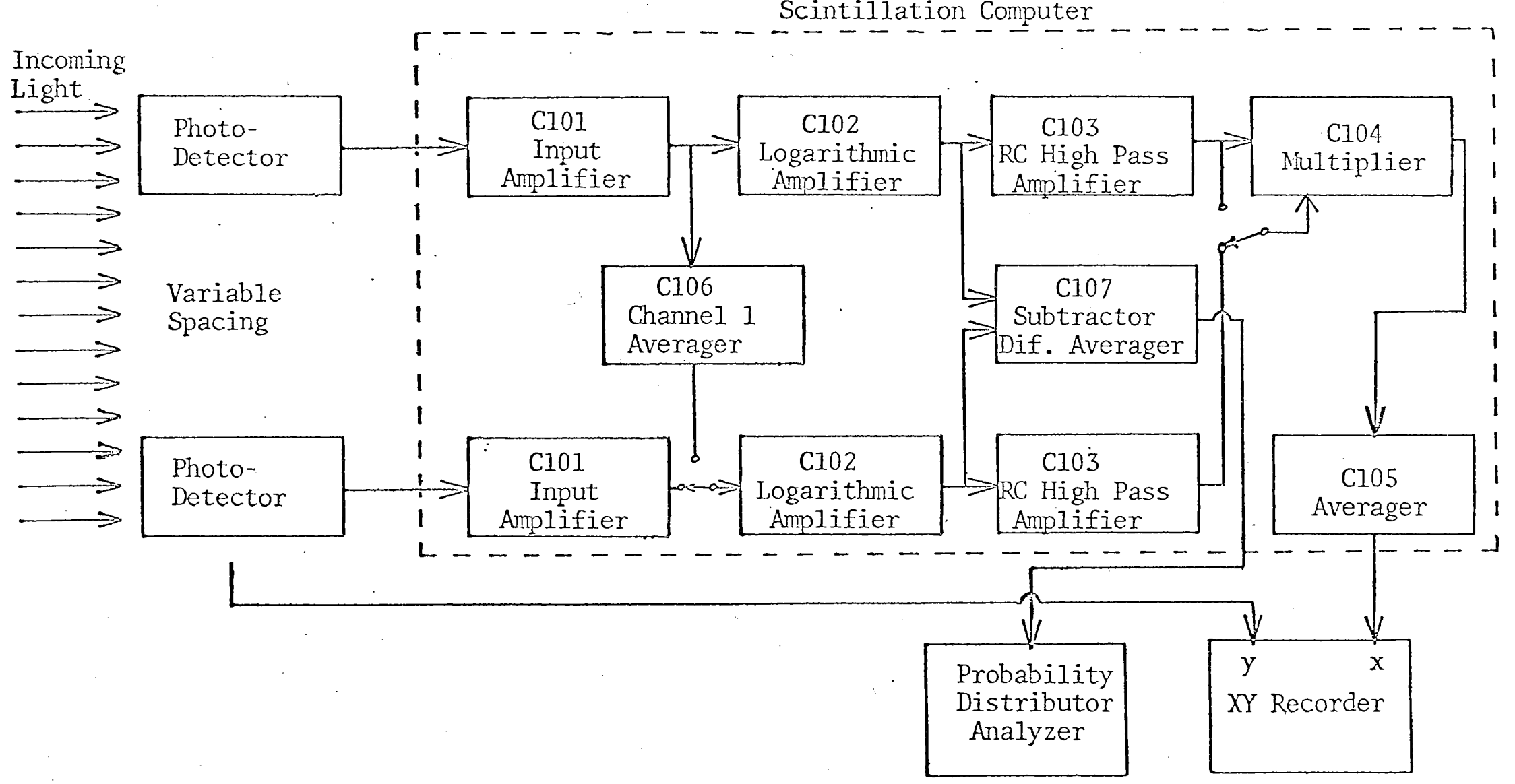

Figure 6. Block diagram of Scintillation Receiver 
Scintillation Computer

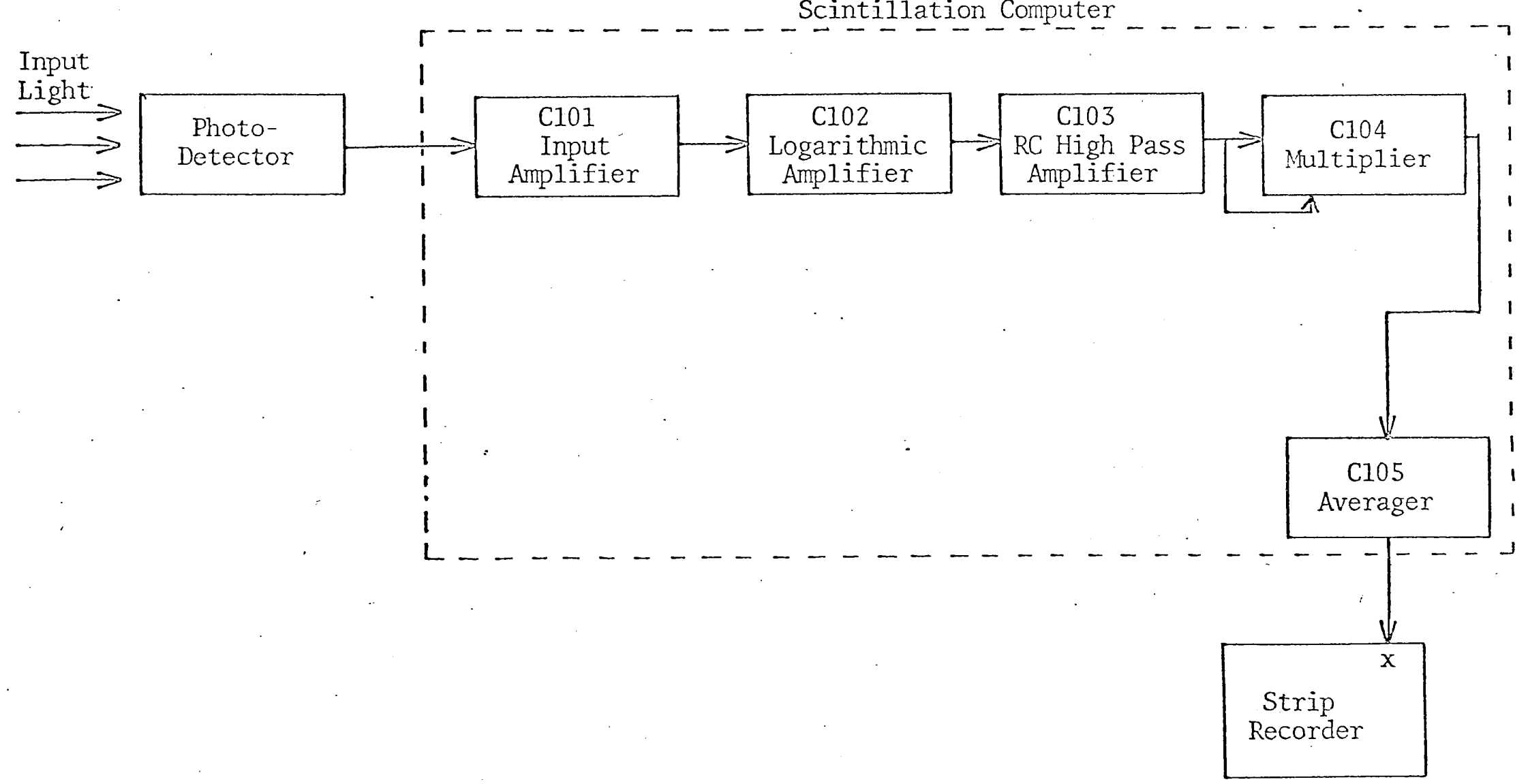

Figure 7. Logic flow for computation of variance. 
Scintillation Computer

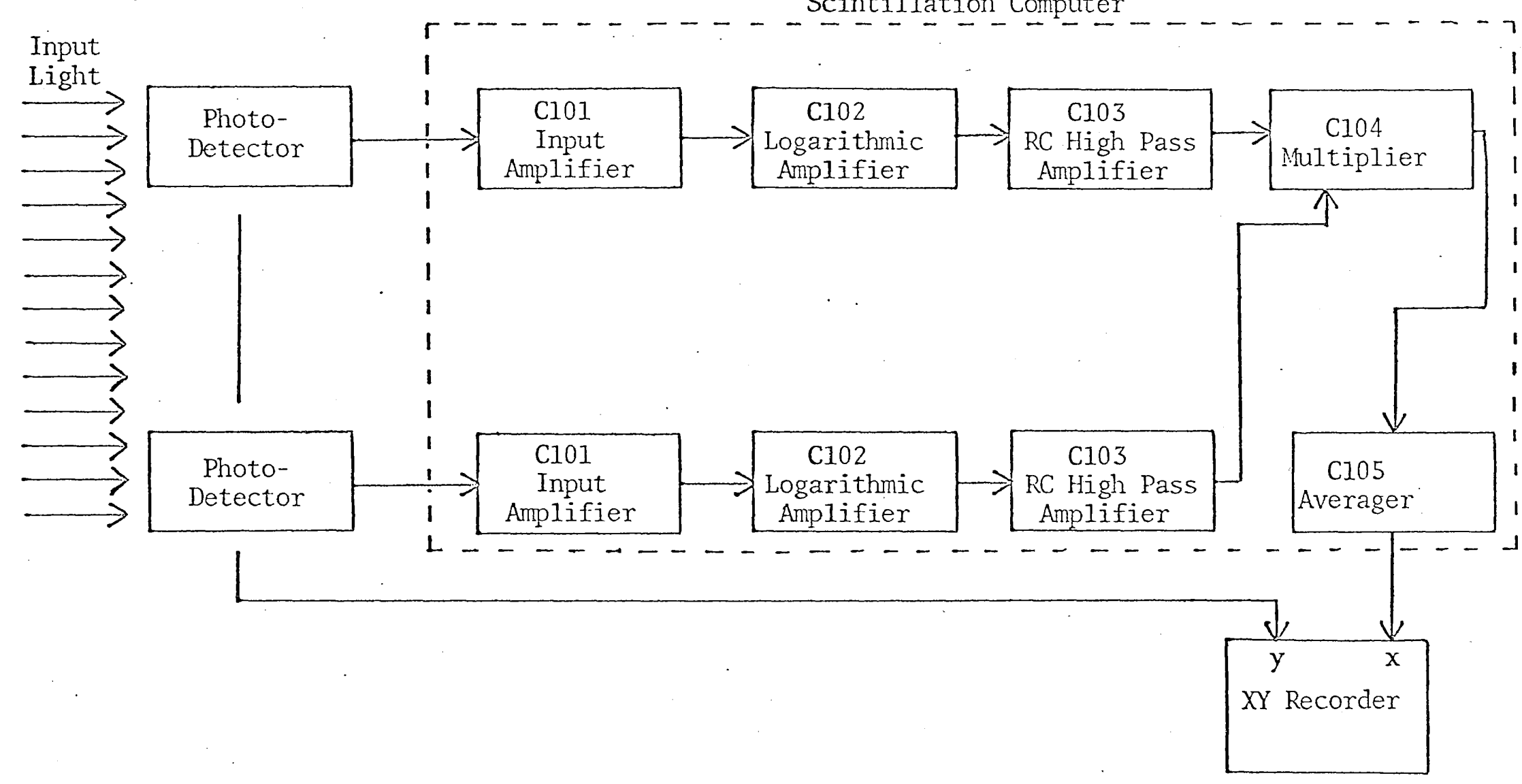

Figure 8. Logic flow for computation of covariance. 
The computation of $\ell$ can be made simultaneously with the computation of variance in order to facilitate investigations of Equation 10.

In order to investigate the probability distribution of $\log$ amplitude $\ell$, we utilize the Probability-Distribution-Analyzer which is swept by the sawtooth output of an oscilloscope (Figure 9). The output is recorded on an $X Y$ recorder.

It is also of interest to examine the spectrum of scintillations, and for this measurement the output of the first amplifier C101 (Figure 9) is fed directly into a spectrum analyzer oscilloscope and recorded either photographically or with an XY recorder and slow sweep.

In all cases, integration time constants (averaging times) are selectable in accordance with the stationarity of the statistics and the type of measurement being made.

\section{Component Description}

Both Channel No. 1 and Channe1 No. 2 have low-noise, calibratedgain input amplifiers, C101 (Figure 10) which are non-inverting buffer units with the gain read on a 10-turn dial on the front panel. The inputs have four possible selections: ground, photomultiplier (or any -.001 to $-5 \mathrm{v}$ signal), unity reference, and decade reference source. The ground position is utilized for offset adjustment (zeroing) of the unit and the decade reference sources are used to calibrate the following logarithmic units. The photomultiplier is the "compute" mode. The reference source voltage is established by adjustment of a front panel potentiometer labeled "unit source" (Figure 10).

The input amplifiers are followed by the logarithmic amplifiers (Figure 11) C102. These units are Philbrick Type SPL4A-P Compensated 
Scintillation Computer

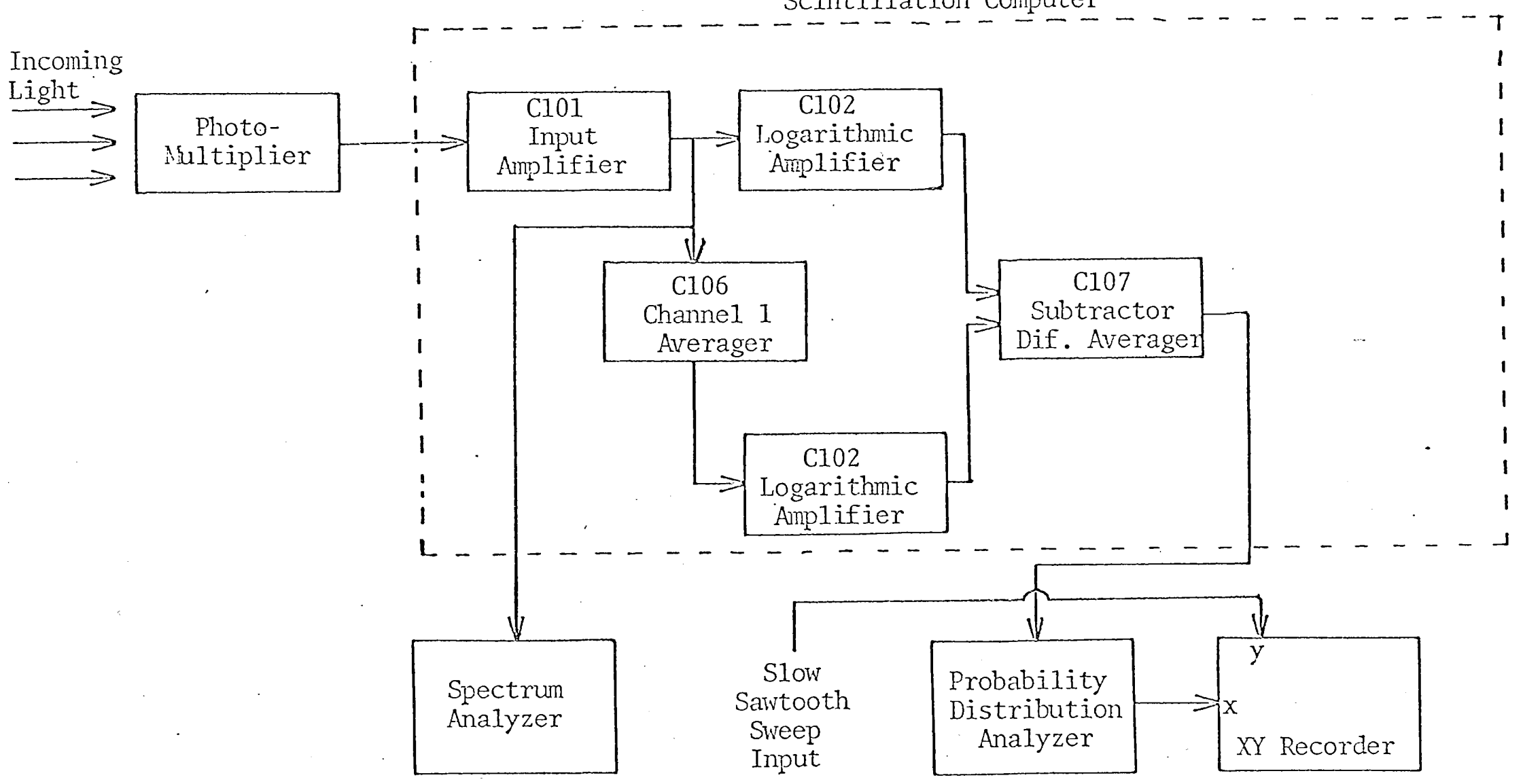

Figure 9. Logic flow of probability distribution analysis and spectrum of scintillations 


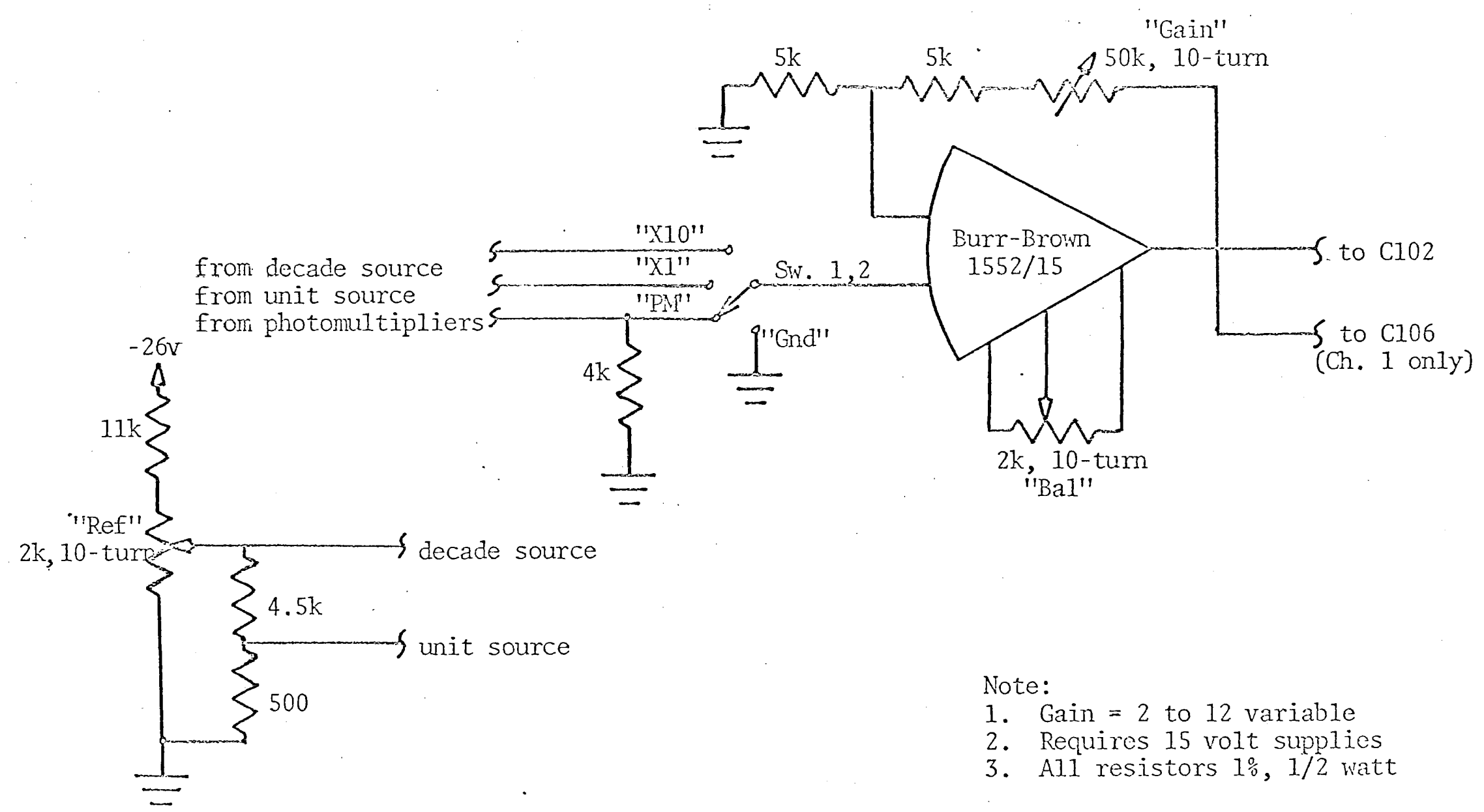

Figure 10. C101, Input Anplifier and Reference Sources 


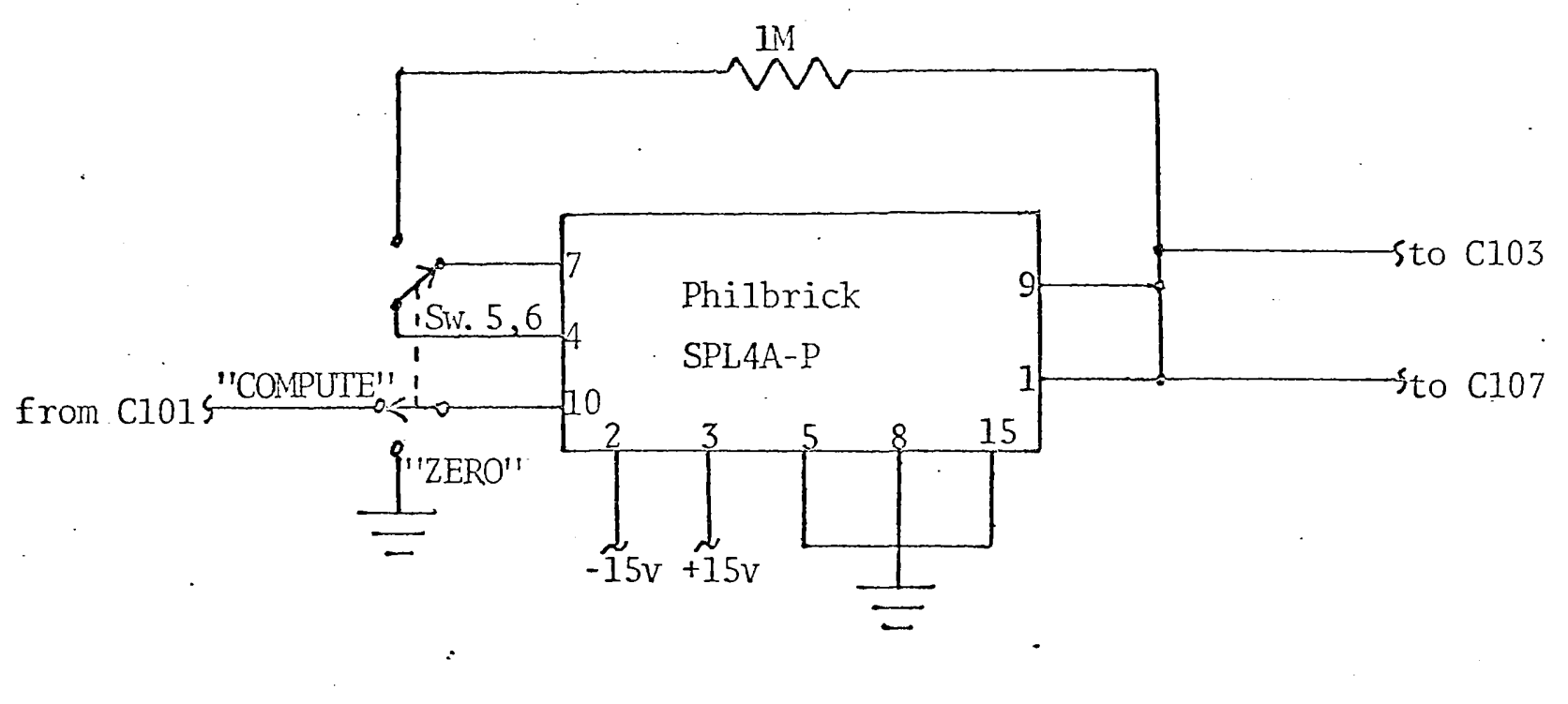

Note:

1. Log unit requires 3 front panel screwdriver adjusts: "reference," "gain," and "zero"

2. Sw. 5, 6 for amplifier zero

3. Requires 15 volt supplies

Figure 11. C102, Logarithmic Amplifier 
Logarithmic Amplifiers, which contain a solid state operational amplifier with a matched logarithmic feedback element. The amplifiers have screwdriver adjustments for amplifier zero, reference adjust, and gain adjust; and one external switch which is used in the amplifier zeroing procedure. In the zero position, this switch connects a 1 megohm feedback resistor to the summing junction while grounding the input, thus producing a X100 inverting amplifier with a shorted input. For compute operation, this switch connects the input resistor to the input amps and connects the active logarithmic feedback element to the summing junction while disconnecting the 1 meg feedback resistor.

The outputs of the $\log$ units feed the RC blocking amplifiers C103; the subtractor-integrator C107; and the multiplier. The RC blocking amplifier is a series capacitor-resistor high-pass filter with selectable time constants as shown in Figure 12. It also contains a non-inverting voltage follower used as a high-input-impedence buffer amplifier. The blocking amplifier is included to give logA- $\overline{\log A}$ which is used in the variance-covariance computation. The balance control can be used to zero this amplifier or to compensate for input offsets on the multiplier which follows.

The multiplier C104 is a Burr-Brown Corp. Mode1 1671/16, Quarter Square Analog Multiplier (Figure 13). The inputs to the multiplier are Channel No. 1 to $\mathrm{x}$ and Channel No. 2 to $\mathrm{y}$ for covariance or Channel No. $I$ to $x$ and $y$ for variance (Switch No. 9). The output from the multiplier is averaged by $\mathrm{C} 105$, referring to Figure 14, which is an inverting amplifier with an RC parallel feedback. The feedback and input resistors can be switched to vary the integration time constant and 


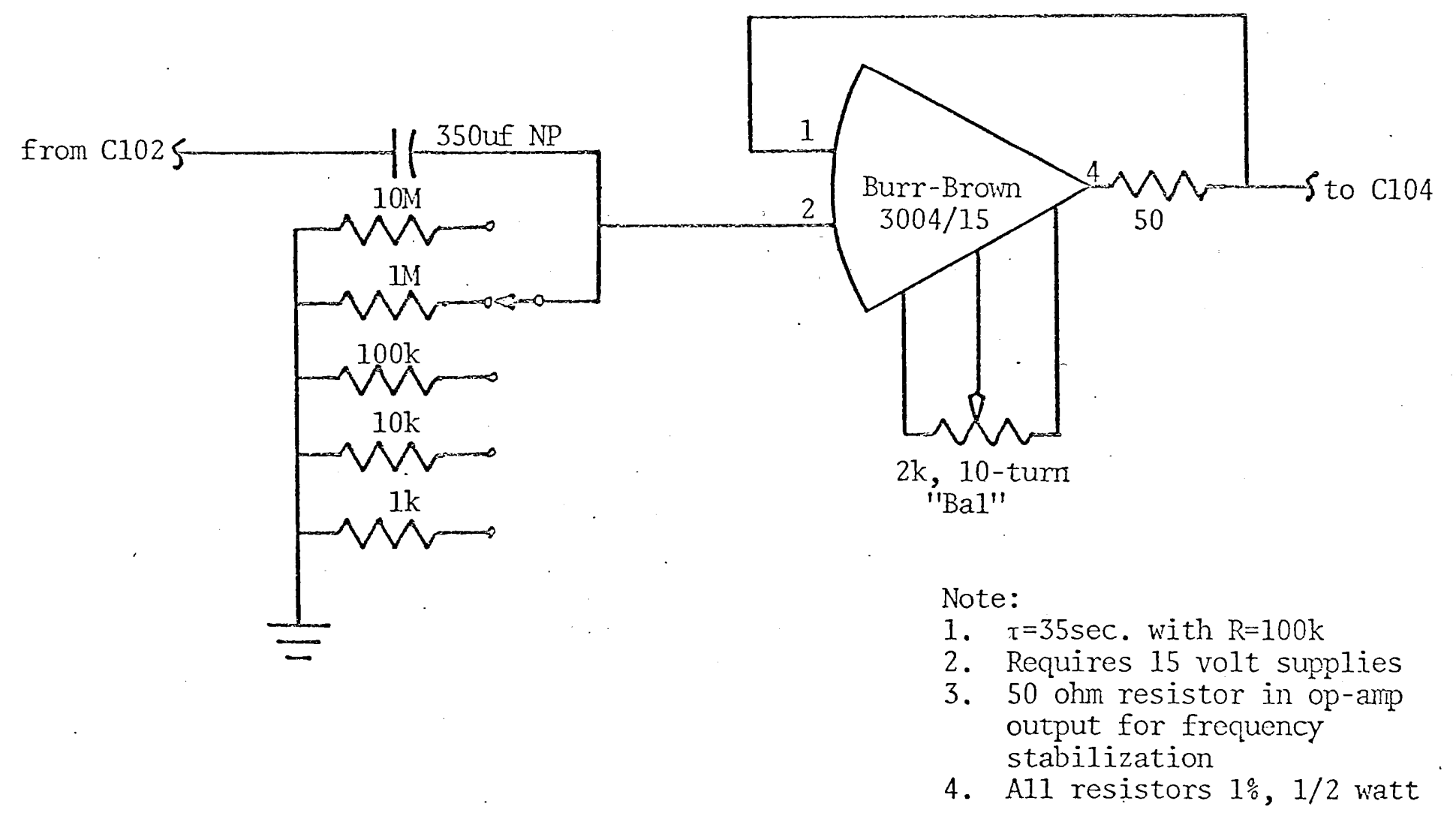

Figure 12. C103, High Pass Amplifier 


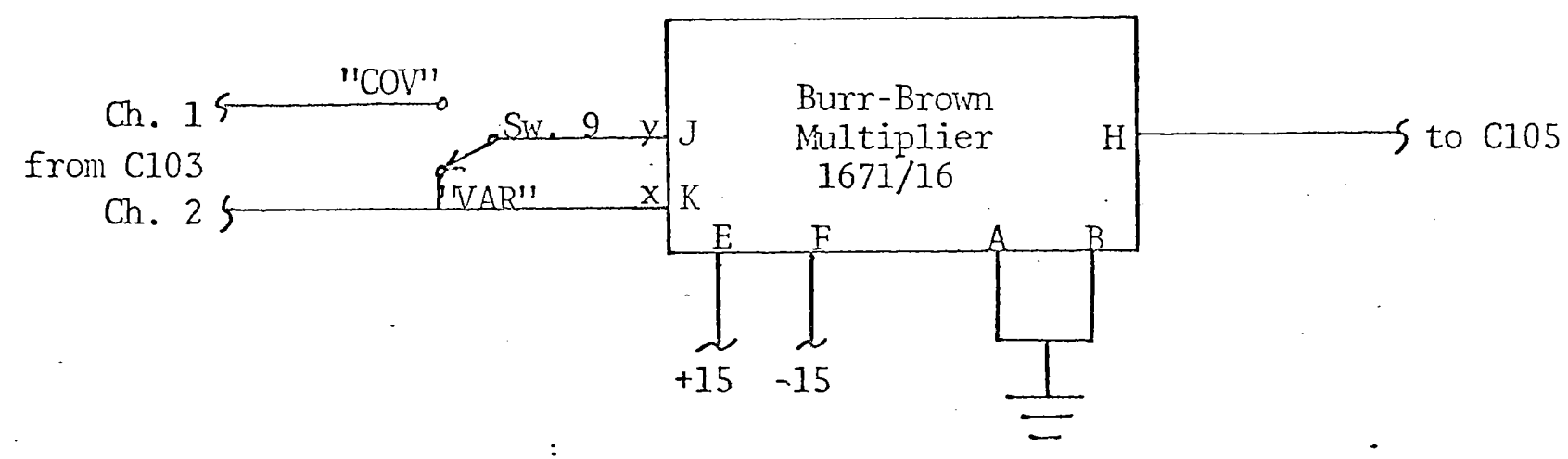

Note:

1. 15 volt supplies required

2. Function switch in position "A"

Figure 13. C104, Multiplier 


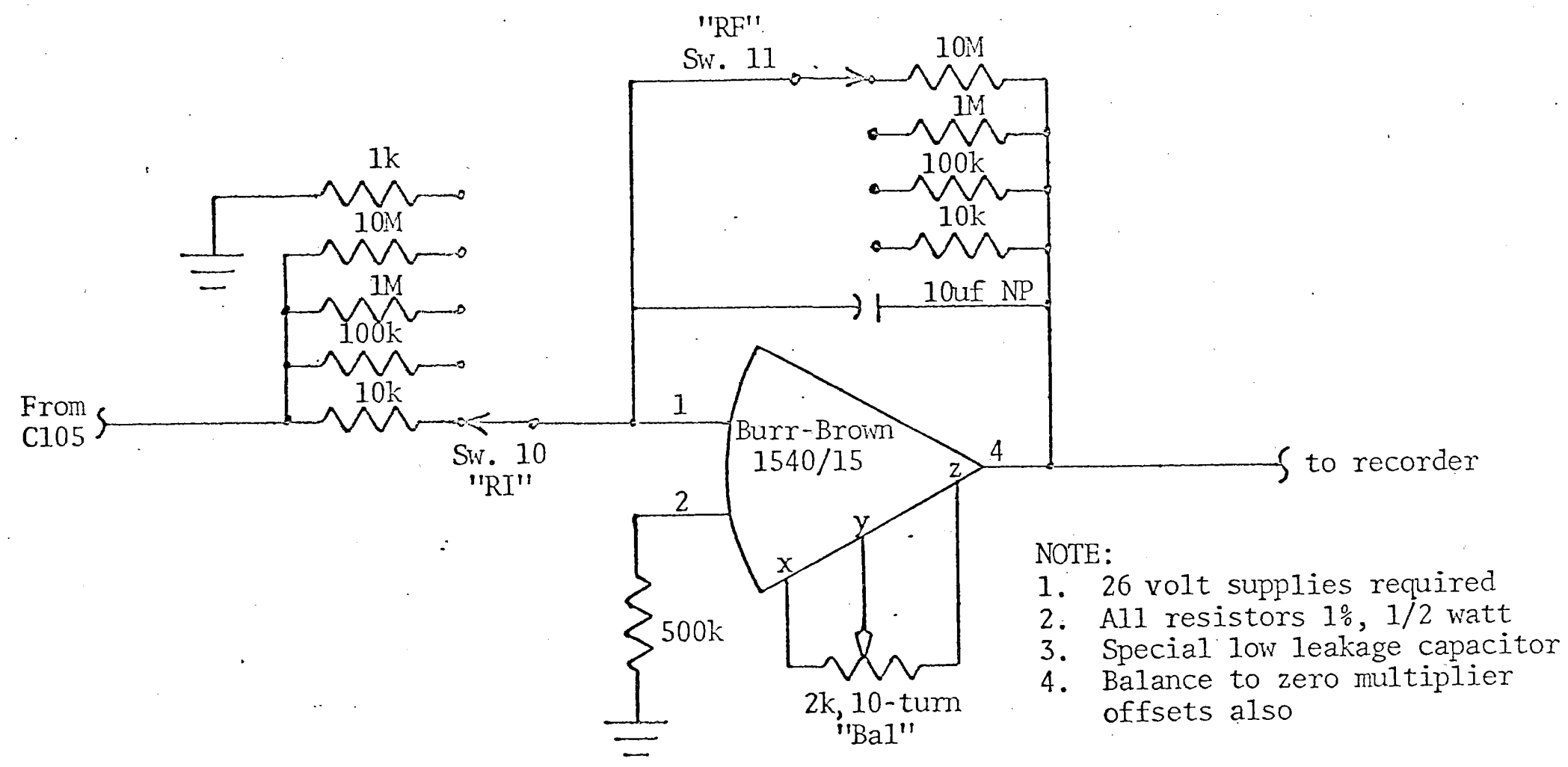

-Figure 14. C105, Averager 
amplifier gain. The output of $\mathrm{C} 105$ is thus the variance or covariance, depending on the position of Switch No. 9.

C106 is a X1, non-inverting, RC parallel feedback averager with selectable time constants (Figure 15). The input and feedback resistors are ganged together and matched for unity gain. It is used between the Channel No. 1 input amplifier and Channel No. 2 log amplifier to provide $\bar{A}$, and is used for computation of $\ell=(\log A-\log \bar{A})$. C107, which is also used in this computation, is a subtractor or differential averager with ganged input resistors, ganged feedback resistors, and ganged feedback capacitors (Figure 16). The feedback capacitors may be switched in for differential averaging or switched out for instantaneous subtracting. Switching input and feedback resistors separately allows choice of time constant and gain.

Oven and Power Supplies

The computer is encased in a metalic oven for shielding from external pickup and for temperature stability (Figure 17). The external requirements for the computer are $110 \mathrm{v}$ ac for the oven heater and $\pm 26 \mathrm{v}$ for the electronics. The outside dimensions of the computer are 22 inches wide by 10 inches high by 22 inches deep. The oven is a double chambered aluminum box with fiberglass insulation inside and out. The larger chamber, which contains the electronics, is separated from the heater section by an aluminum partition with two shielded air passages. The partition isolates the 60-cycle heater supply from the electronics. The heater includes a fan, a heater element, and an electronic temperature control with thermistor-sensing which is 


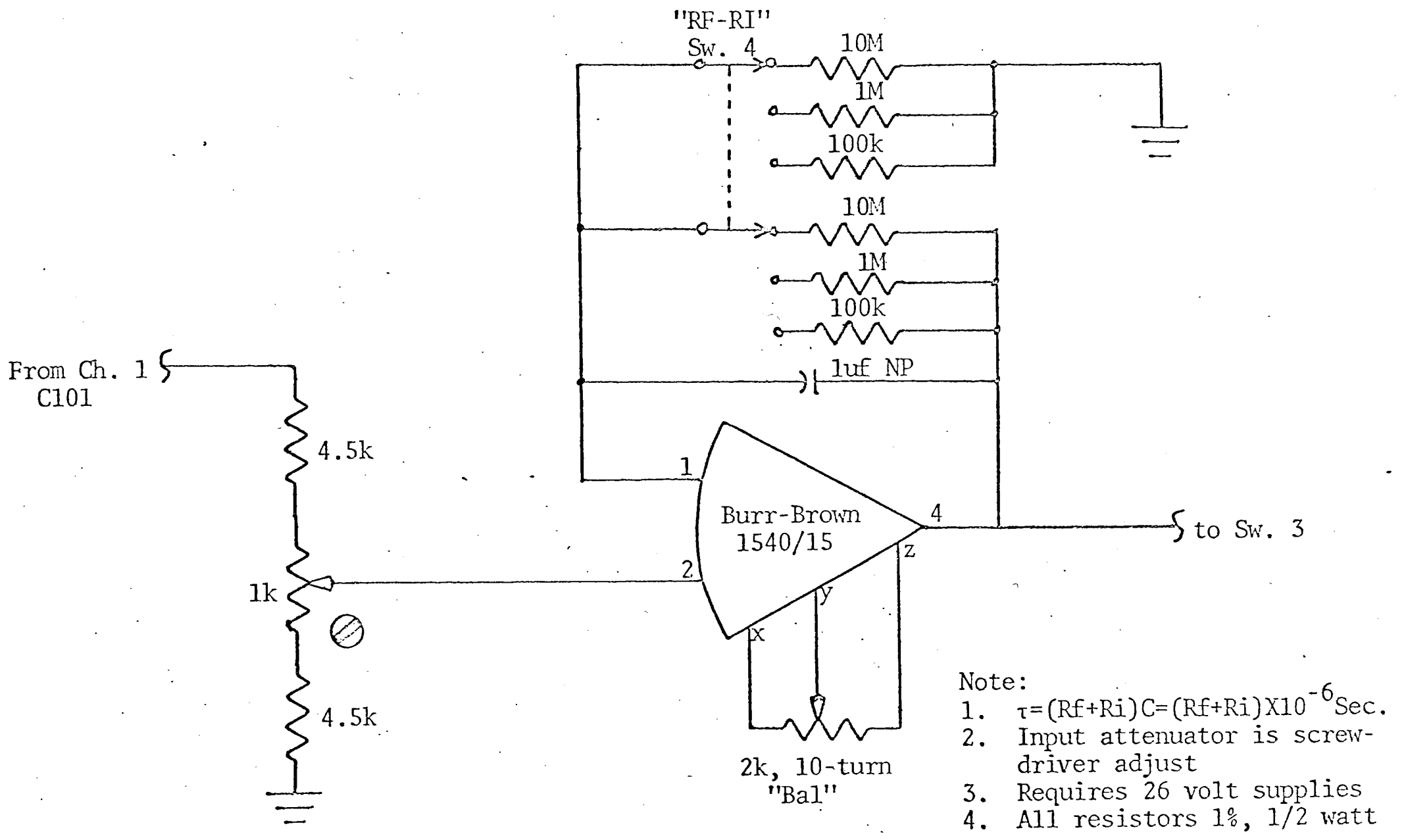

Figure 15. C106, Channel No. 1 Averager

N 


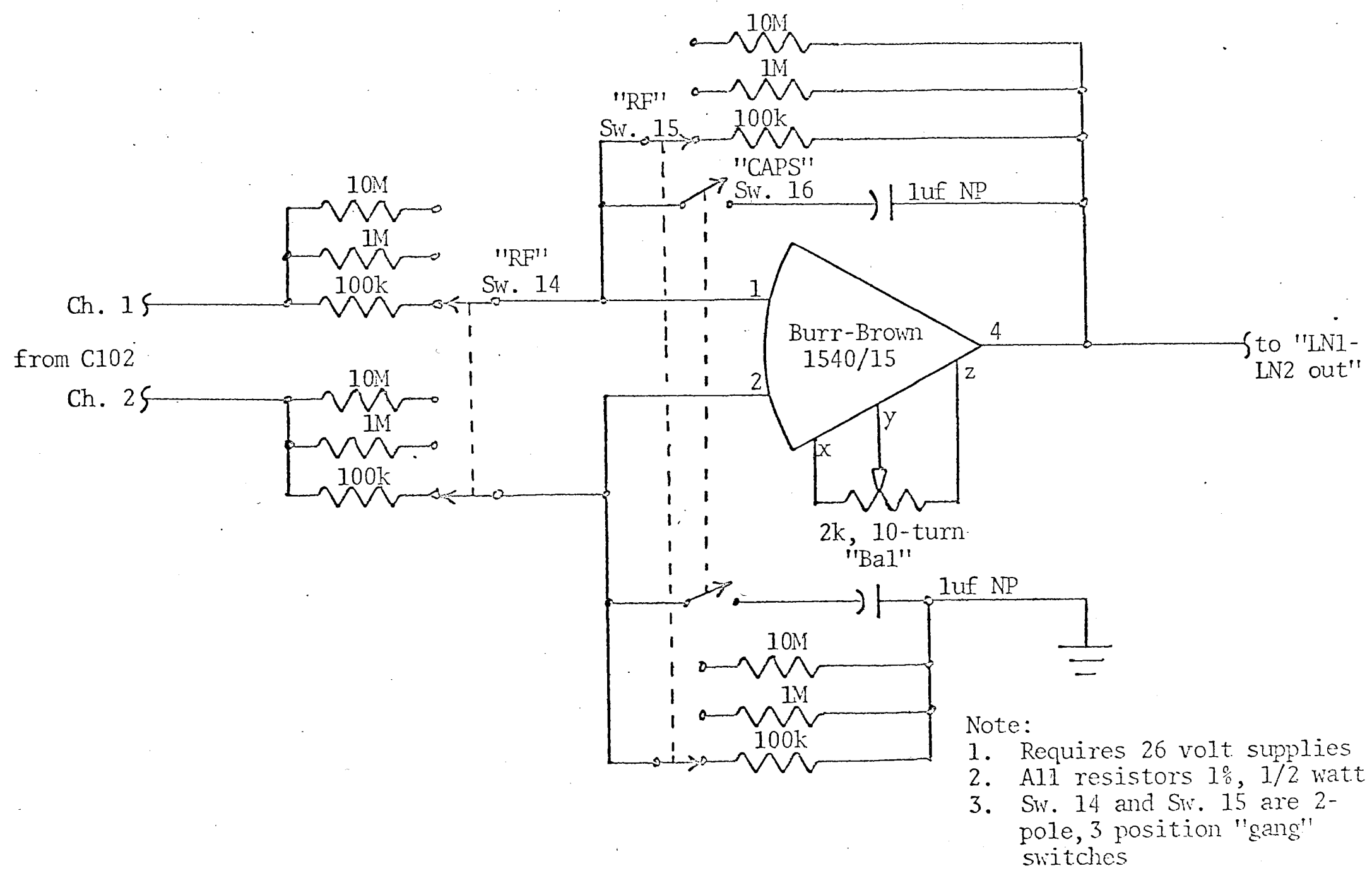

Figure 16. C107, Subtractor-Differential Averager 


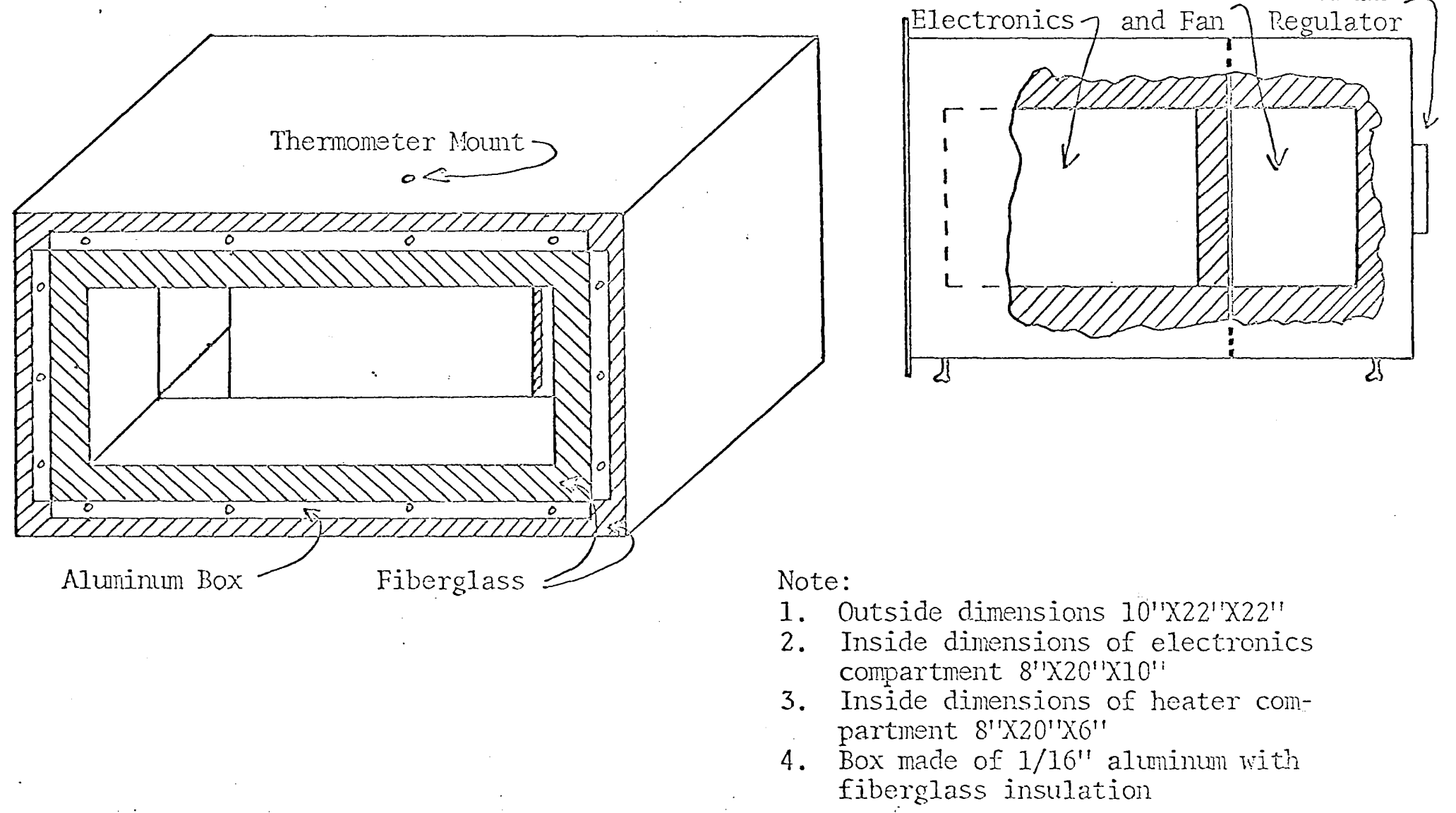

Figure 17. Isometric drating of Gren 
designed for less than $0.1^{\circ} \mathrm{C}$ temperature drift (Figure 18). The sensor is attached rigidly (and thermally) to one of the aluminum heater exit deflectors to improve its response time and control the heater exit temperature. The heater is designed to raise the air temperature in the oven to $37^{\circ} \mathrm{C}$ and then hold it constant at that temperature.

Since the offset voltages of the individual amplifiers drift with changes of temperature, it is quite important that the oven temperature maintain a stable equilibrium. Once the oven and electronics have reached equilibrium, all offsets are balanced out using the trimpots. Hence, the exact equilibrium value is not important but rather the stability of that equilibrium temperature. During the initial calibration, it was observed that the temperature regulation of the oven was sufficient to eliminate observable drift.

While most of the internal electronics require 15-volt supplies, some of the output amplifiers require 26 volts. Therefore, the computer contains its own 15-volt voltage regulators which supply the 15 volt components from the 26-volt external supplies. The 15-volt regulator is a series regulator utilizing a power transistor driven by a differential amplifier as shown in Figure 19. It may be adjusted from 13 volts to 16 volts, but also contains an over-voltage protection circuit which turns on a transistor switch if the supply exceeds 15.1 volts. This shunts large amounts of current until the supply drops to 15 volts, and is designed to protect against both power supply failure and operator error. Since the Trygon power supply used for the 26-volt source includes a current limiting feature, the over-voltage circuit 


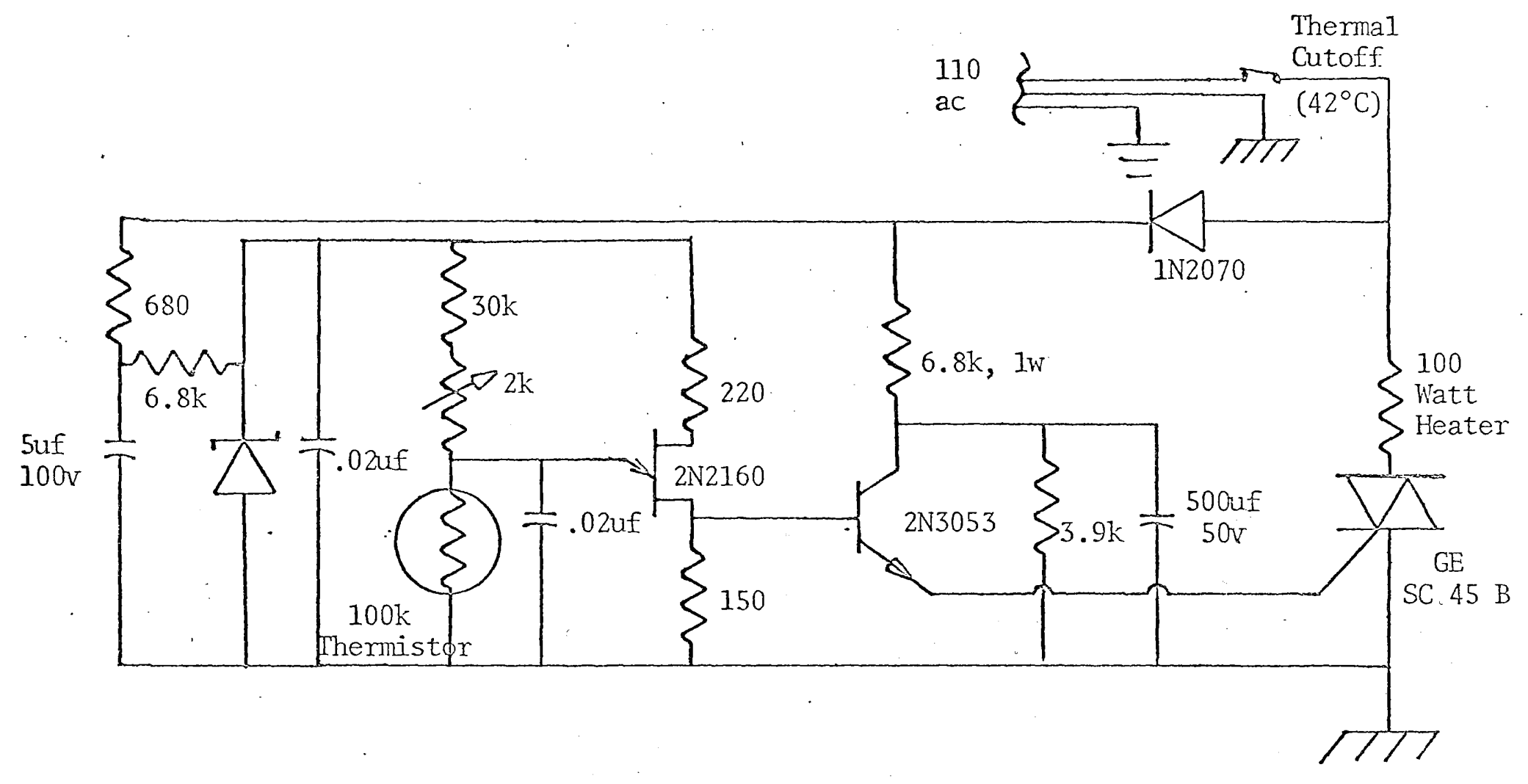

Figure 18. Thermal regulation circuitry 
to $26 \mathrm{v}$ electronics

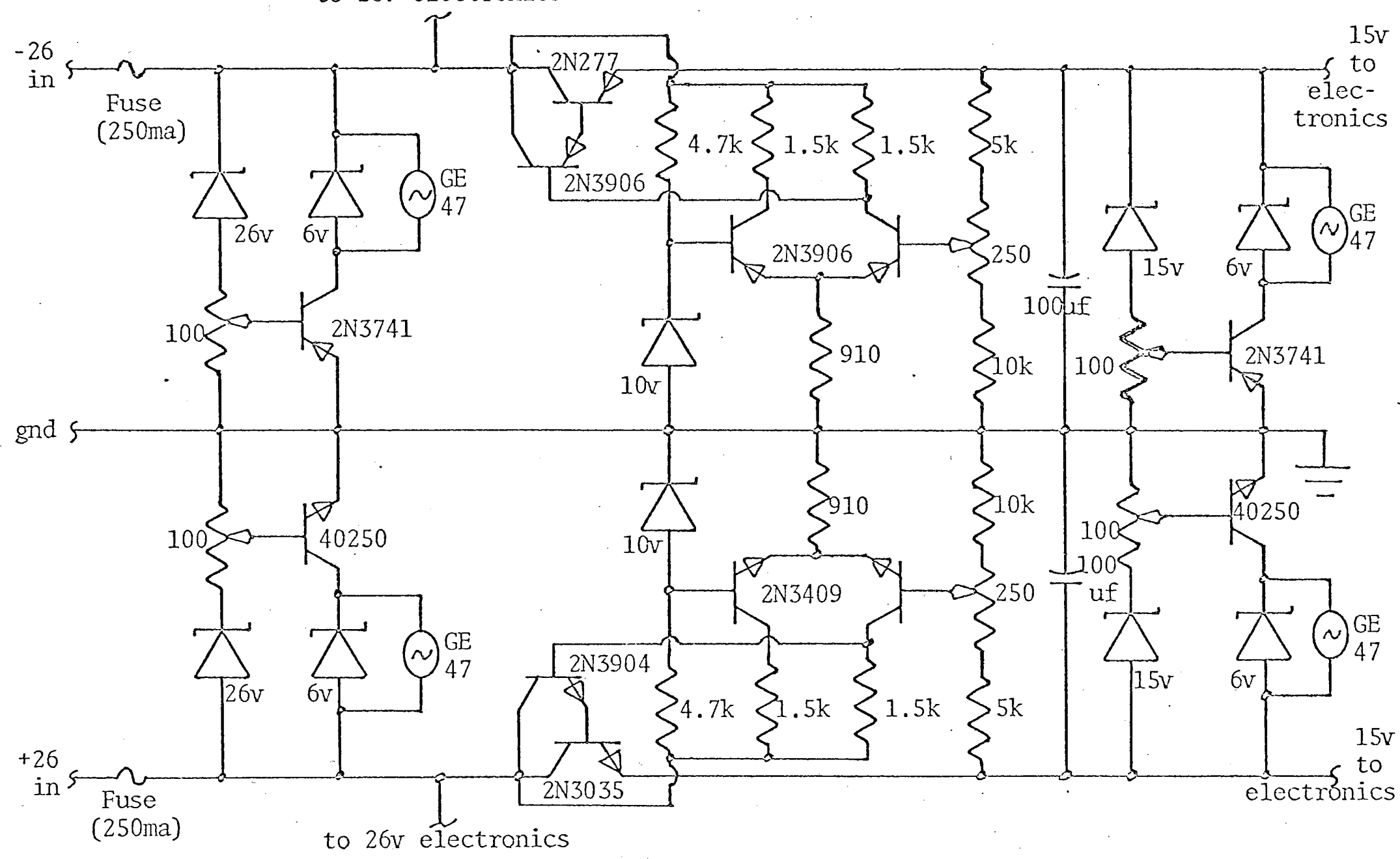

Figure 19. Internal 15 volt regulator with over-voltage protection circuit 
will draw this supply down without damaging the supply or the electronics of the computer. If the Trygon supply should fail to currentlimit or if the over voltage is too fast for it to respond, the sudden current surge will blow the fast-blow fuses which are used to back up the current limiting safety feature of the Trygon. This over-voltage protection is also incorporated into the 26 volt supply input to the computer.

Bandwidth, Dynamic Range, and Calibration

The bandwidth of the log covariance calculation is limited by the $10 \mathrm{kHz}$ (1 per-cent error) specification of the Burr-Brown Corp. ModeI 1671/16 multiplier, since all other amplifiers including the logarithmic amplifiers have $1 \mathrm{MHz}$ bandwidths. The maximum fluctuation rate of the atmospheric turbulence is about $1 \mathrm{kHz}$, and therefore $10 \mathrm{kHz}$ is sufficient bandwidth.

There are two separate types of dynamic range which must be considered: first, the dynamic range of the acceptable input signal level, i.e., large signal fluctuations which instantaneously approach zero and which feed into log units; and secondly, the dynamic range of the output signal, i.e., small fluctuations yielding covariance values approaching zero. The input dynamic range is established by the four decade dynamic range of the logarithmic amplifiers since the input amplifiers easily exceed this four decade specification. Care must be taken to eliminate ground-1oop currents and noise pickup. The output dynamic range is limited by the Burr-Brown multiplier; however, although the multiplier's specification lists the error signal including drift and offset as being "less than 1 per cent of full scale," 
i.e., 0.1 volt, we found that by balancing the RC blocking amplifier to compensate for the offsets in the multiplier we were able to obtain better than three decades of dynamic range.

Calibration of the computer was completed in two steps: the gains, offsets, and reference voltages were tested and set to their proper values; and then the computation process was tested with known electrical and optical input signals. The latter process is complicated by the logarithmic response to very small voltages.

A $(-1 \mathrm{mv}$ to -1 volt $)$ square wave was used to test the calibration of the variance and covariance modes, as follows. First, the signal was applied to one channel (covariance mode) and an unrelated signal and a zero signal were in turn applied to the other channel, while the output was observed to remain at zero. Second, the $1 \mathrm{mv}$ to 1 volt square wave was applied to both inputs with an input gain of X10. The output of the log unit was observed to be $a+3$ to -6 volt square wave, having been calibrated for $3 \log _{10}\left(10 \mathrm{~V}_{\text {in }}\right)$. This results in a 9 volt (peak to peak) square wave out of the RC blocking amplifier and $(4.5)^{2} / 10$ volts out of the multiplier, which with a $X 1$ averager yields 2.025 volts.

After verifying these relations, Switch No. 9 was changed to the variance position and it was verified that variance equals covariance when the inputs are identical.

Following this, the computation of $\log 1-\log 2$ (i.e., the output of C107) was tested with the same square wave input. First, it was verified that the output of $\mathrm{C} 107$ was zero with common inputs to both of the log amplifiers. Then, the input to the Channel No. 2 log 
amplifier is switched to ChanneI No. I average (Switch No. 3) such that its output should be $3 \log _{10} 5$ or +2.1 volts. In the subtract mode, C107 should yield $a+.9$ to -8.1 volt square wave, and in the average mode it should yield a -3.6 volt signal out. This was verified and then the input to Channel No. 1 was changed to a -1 volt signal which again resulted in a zero output signal.

The amplifier offsets were then checked and the input signal was changed to a 0.05 volt peak-to-peak wave riding on a -0.5 volt dc bias, which was used for small-signal testing. The gain of the output averager $\mathrm{C} 105$ was set at $\mathrm{X} 1$, and the output observed. A variance and covariance of $3.8 \mathrm{mv}$ was expected, and the observed answer was $3.7 \mathrm{mv}$. The square wave was then changed to 0.005 volts peak-to-peak, which yields an expected output of $0.0042 \mathrm{mv}$; however, the output here was $0.27 \mathrm{mv}$. The discrepancy is attributed to multiplier errors, and establishes a limit of better than three decades of useful output signal (i.e., 10 volts to $10 \mathrm{mv}$ with 1 per cent error).

This entire procedure was repeated using a laser, a chopping wheel, and a photomultiplier to verify that the computations remained valid when interfaced with the optical system.

\section{REPRESENTATIVE RESULTS}

In this section experimental curves are presented as examples of computational results. These curves as yet have little quantitative significance in that no meteorological data were collected. Final quantitative data will be taken in the near future on a more uniform 
path with supplemental meteorological instrumentation, under a grant from the Office of Naval Research.

The first two curves are of the covariance of the log amplitude as a function of the separation $(r)$. The curve in Figure 20 was recorded at 12:15 p.m. on a cool, overcast, calm day and shows a $10 \mathrm{~g}$ amplitude correlation distance of approximately three inches. It also shows a "tail" or reoccurance of positive covariance at about seven inches separation. This can be explained by the non-uniformity of the path (11). The second covariance curve, Figure 21, was recorded later in the same day, and shows a much shorter correlation distance with larger fluctuations. This is attributed to the normal increase in turbulence in the afternoon. If these short correlation distances are found in future experiments, over a uniform path, a lens-expansion system will be utilized to scale-up the two or three-inch correlation distance for more detailed examination.

Curves such as those shown in Figures 20 and 21 may be utilized to determine the amplitude correlation area and to deduce the structure of the turbulence by means of a convolution process (13), as well as to verify theoretical predictions based on independent meteorological data.

The third curve, Figure 22 , is of the variance of the log amplitude versus time, showing the gross fluctuations in the variance of $\ell$ at the receiver. The curve was also taken late in the afternoon of June 27,1968 . The choice of averaging or integrating time constants is seen to be complicated under such unstable meteorological conditions. A short averaging time results in noisy curves, e.g., covariance, 


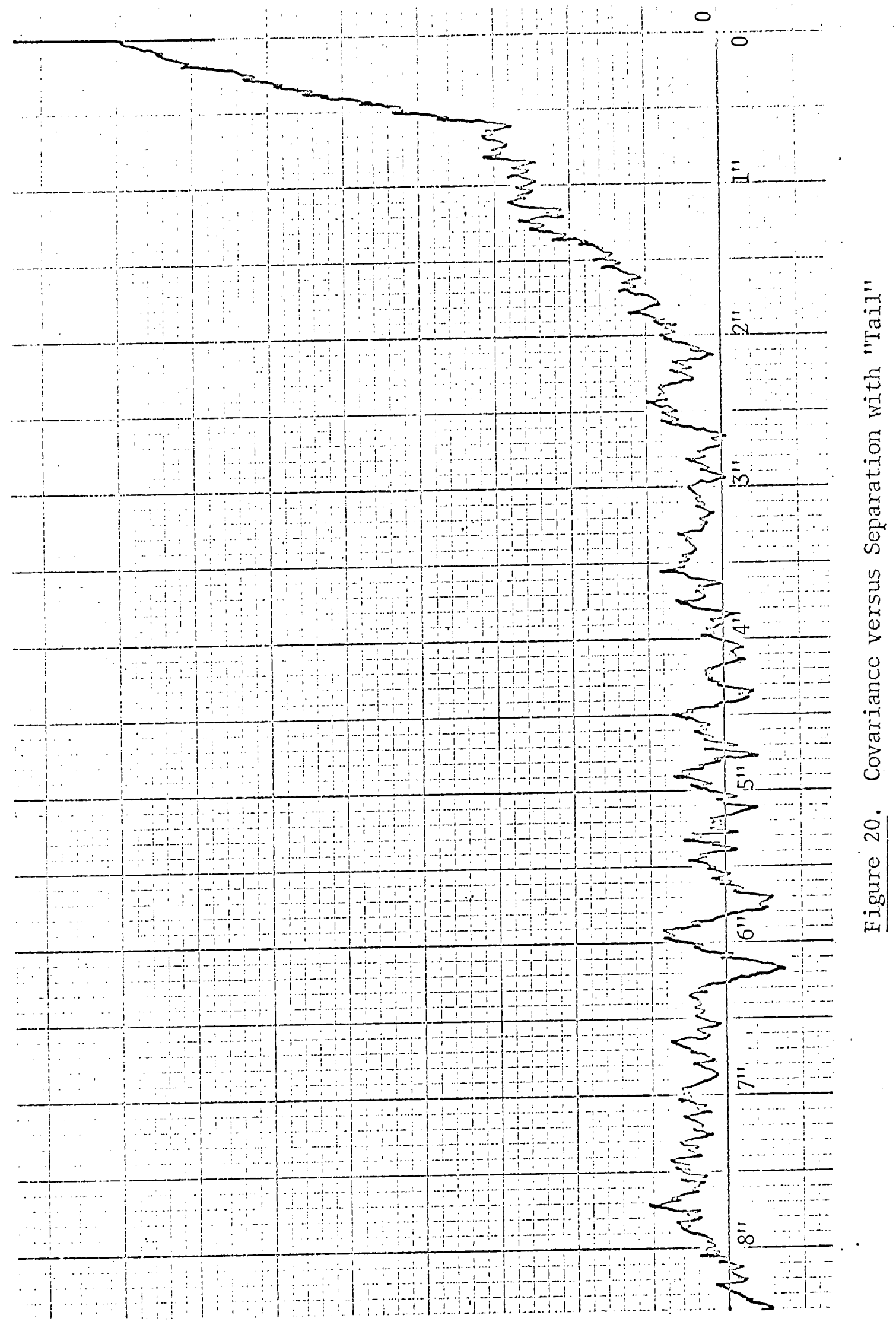




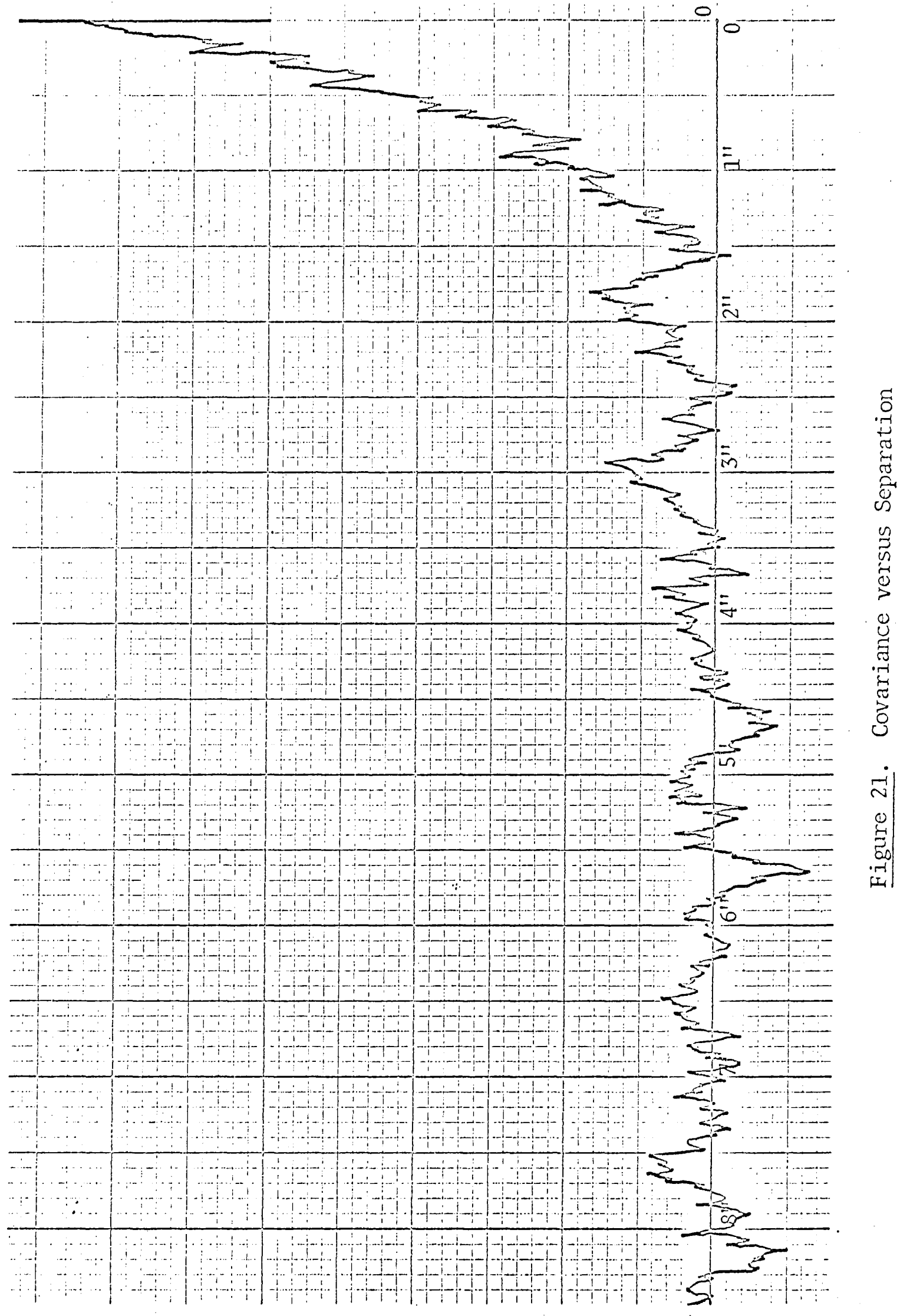




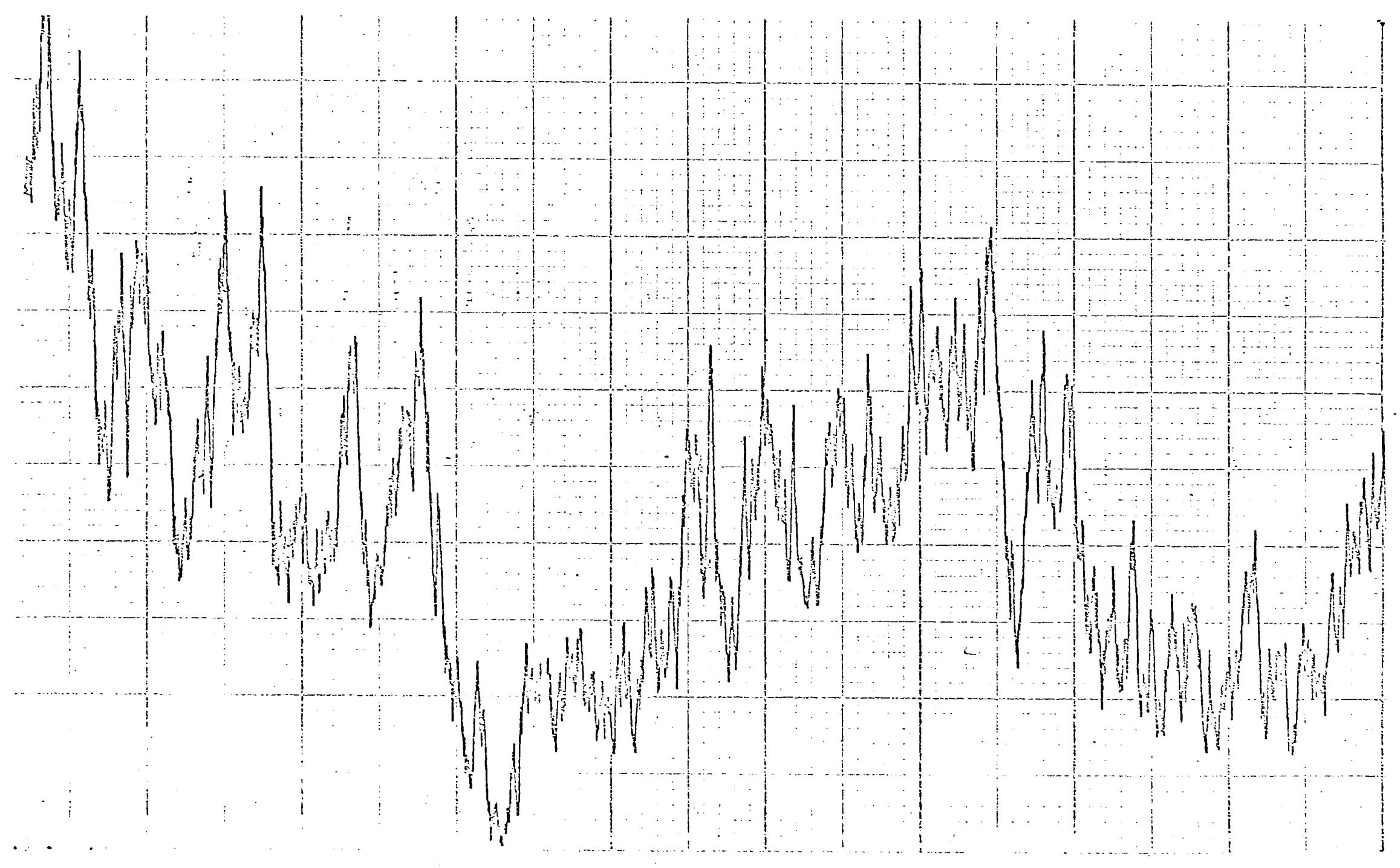

Figure 22. Variance versus Time (2 inches per second horizontal) 
while a long averaging time may result in non-stationary conditions which distort the covariance. If the variance versus time does not indicate an appreciable trend over a few minutes, then long averaging times (such as 60 seconds) for covariance are desirable. This limits the allowable separation rate of the detectors.

The curves of Figures $23 \mathrm{~A}$ and $23 \mathrm{~B}$ are of the probability distribution integral of the amplitude A. The first is recorded as computed by the probability-distribution analyzer and the second is averaged by a RC low pass filter with a time constant of one second. The sweep speed used to drive both readings is five seconds per division. Such a probability curve can be checked for log normality through the use of log probability paper. Alternatively, the quantity $\&$ may be directly checked for normality.

The final curve (Figure 24) is a photograph of the spectrum of amplitude scintillations. The curve was made using a Tektronix Type IL5 Spectrum Analyzer with a center frequency of approximately $50 \mathrm{~Hz}$ and a dispersion of $50 \mathrm{~Hz}$ per $\mathrm{cm}$. with a sweep rate of 0.2 seconds per $\mathrm{cm}$. The peak frequency and rate of fall-off can be compared with transverse wind velocity, amplitude correlation area, and theoretical predictions (3) 

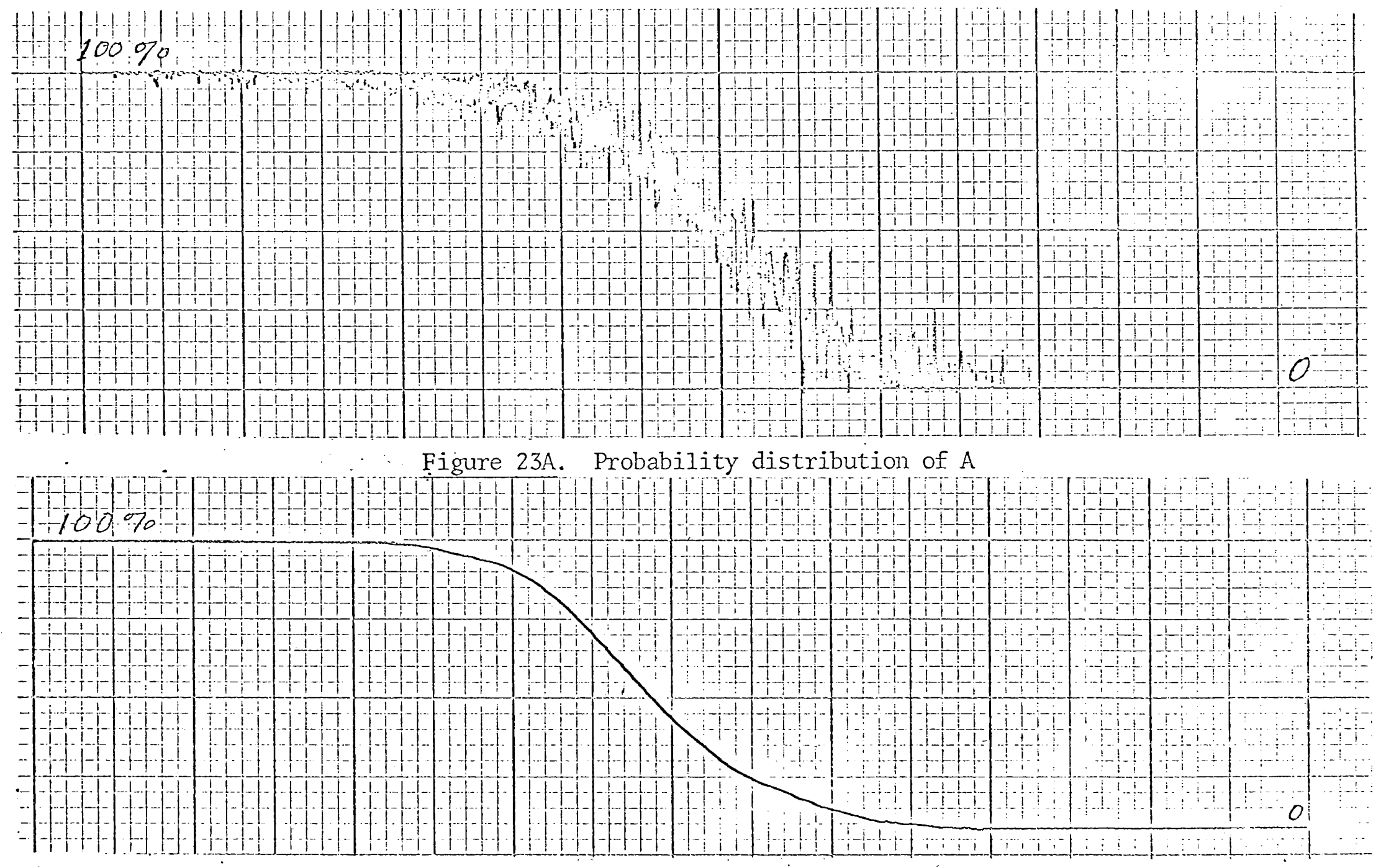

Figure 23B. Probability distribution of A with averaging ( $\tau=1 \mathrm{sec}$.) 


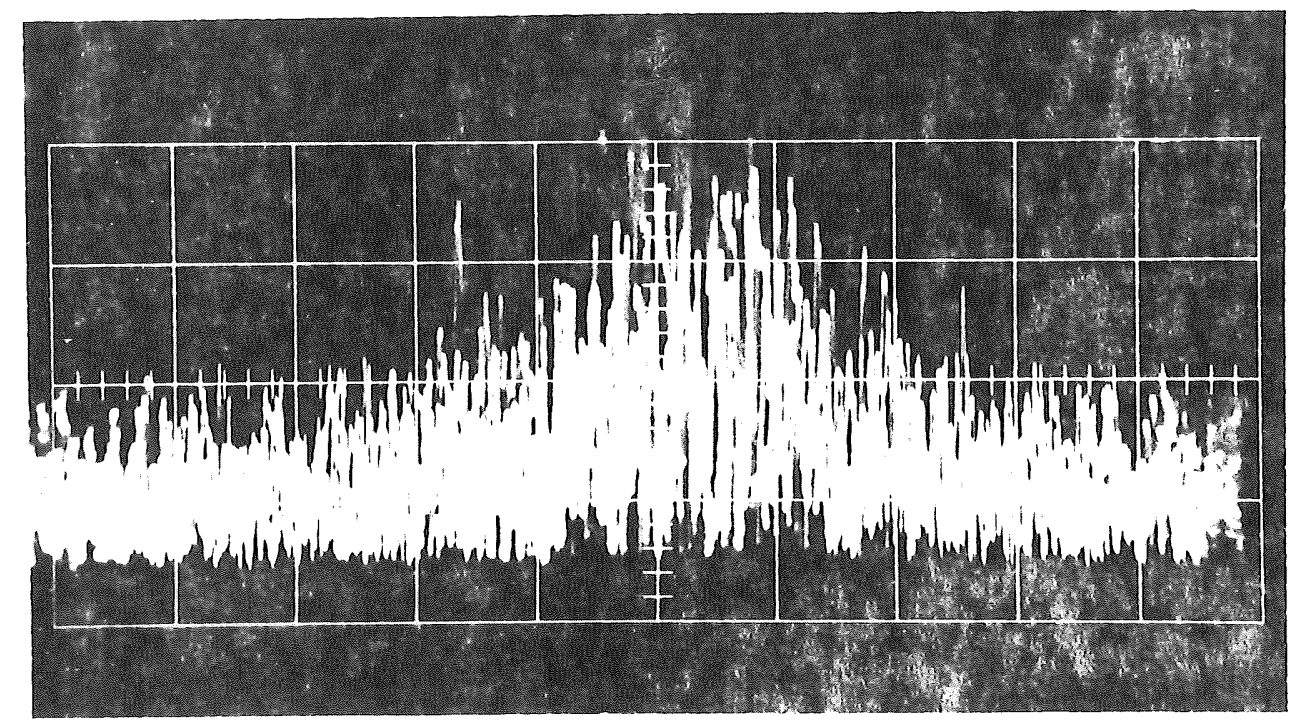

Figure 24. Photograph of spectrum of amplitude scintillations 
CHAPTER III

\section{PULSE DISTORTION SYSTEM}

\section{GENERAI SYSTEM}

The Pulse Distortion System (Figure 25) was designed to detect distortion due to transit-time dispersion during atmospheric propagation, as discussed in Chapter I. The system includes a "mode-locked" laser, the atmospheric path, a wideband detector, a sampling oscilloscope, a frequency-modulated (FM) tape recorder, and the distortion analyzer which has been developed.

The short (two nanosecond) pulses are created by a mode-locked (16), 50 milliwatt, helium-neon, Spectra-Physics Model 125 Laser. The long (two meter) optical cavity allows a number of longitudinal modes (17) to oscillate within the atomic line width of the helium-neon gas. These longitudinal modes are separated in frequency by $c / 2 \mathrm{~L}$, where $\mathrm{c}$ is the speed of light and $\mathrm{L}$ is the length of the optical cavity, and are those resonant frequencies for the cavity which fall within the atomic line shape of the lasing medium. In free-running operation, these modes compete with adjacent modes for power from the medium, which cause instability in the amplitudes of the individual modes as illustrated in Figure 26, and thus also in the total output. When an electro-optic perturbation is placed within the cavity (Figure 2) and caused to oscillate near this $\mathrm{c} / 2 \mathrm{~L}$ frequency (in this case approximately $75 \mathrm{MHz}$ ), then the longitudinal modes oscillate in a 


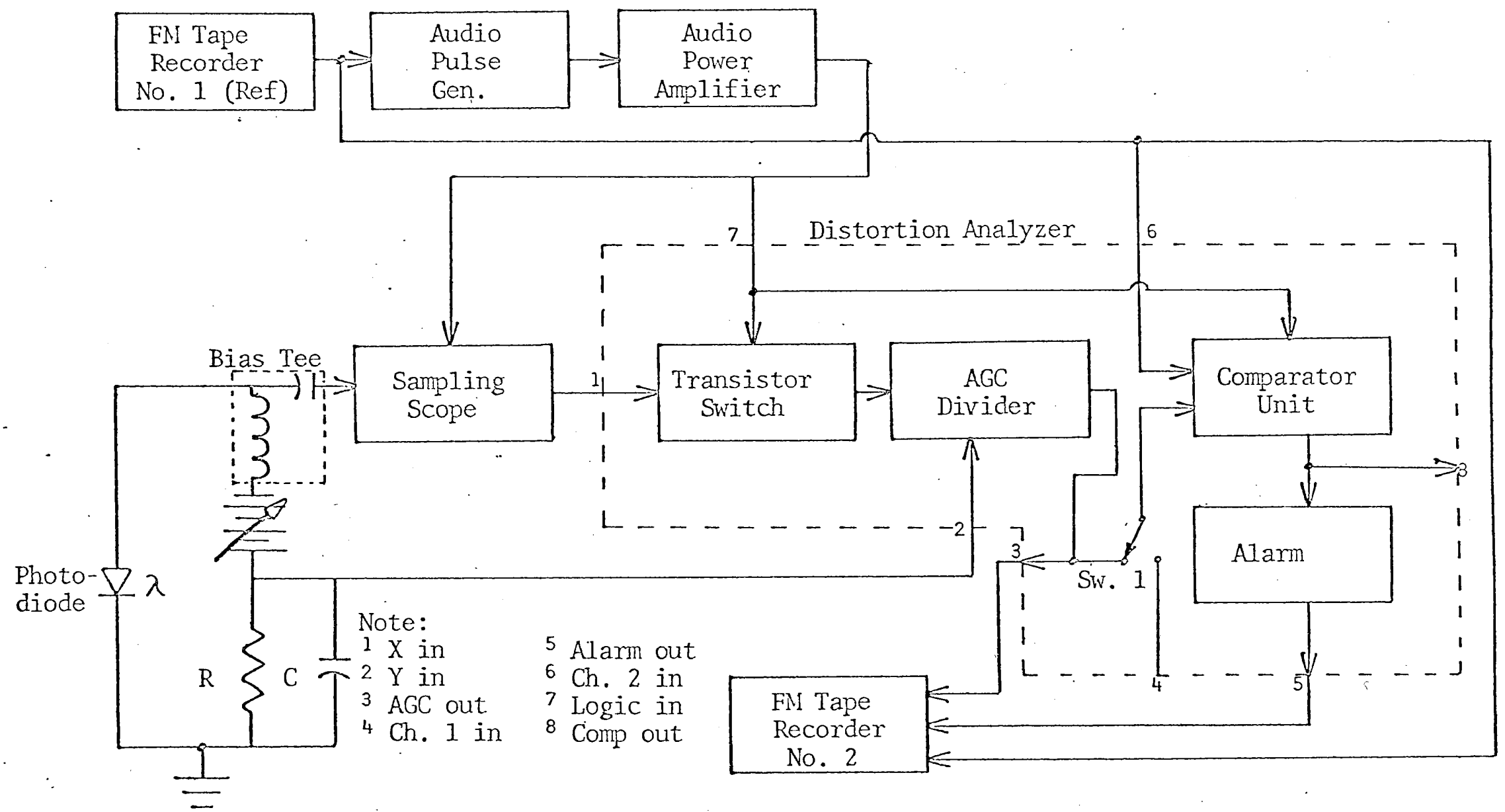

Figure 25. Block diagram of Short Pulse Distortion System 


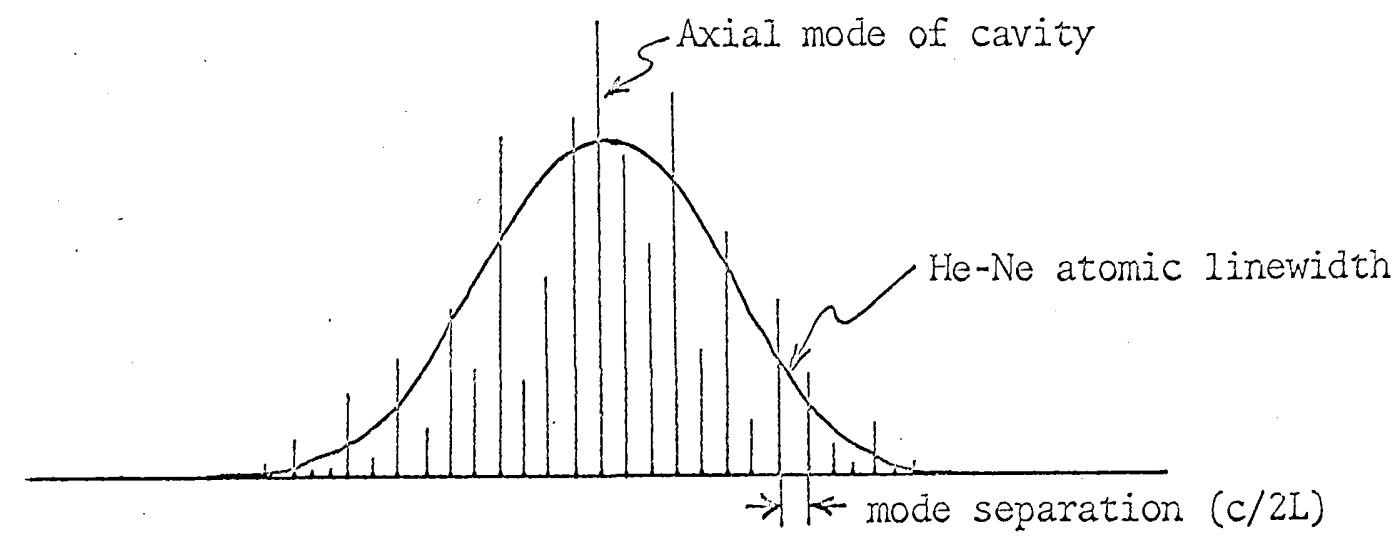

Frequency spectmum of laser oscillation showing irregularity of mode amplitudes due to intermode competition

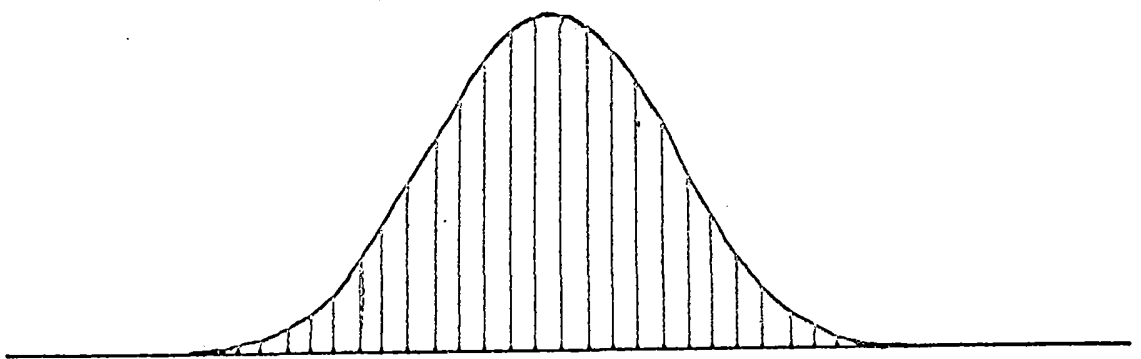

Frequency spectrum of laser oscililation during "mode-lock" showing stability of mode amplitude conforming to atomic Iine shape

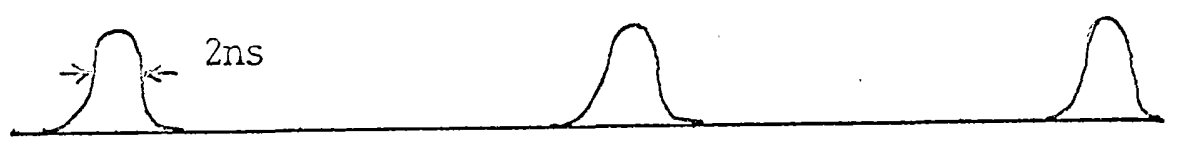

Time domain representation of laser output in "mode-1ock" Figure 26. Diagrams of Iaser mode oscillation 
stable fashion and with relative amplitudes which conform to the atomic line shape as shown in Figure 26. Since the lasing medium has an inhomogeneously broadened line shape (18), it is a gaussian, and therefore the modes constitute a good approximation to the fourier spectrum of short, repetitive gaussian pulses. Thus the output of the laser in the time domain, referring to Figure 26, is a series of short optical pulses with approximately a $75 \mathrm{MHz}$ repetition rate.

The short pulses are transmitted through the atmosphere on a onemile path between the Oregon Graduate Center and our remote site on Skyline Drive. The beam is focused to a one-inch spot at the receiving end through the use of a large, diffraction-limited Cassegrainian telescope at the transmitting end. Also, a large, non-diffractionlimited lens is used to capture all the beam and focus it on a wideband photodiode detector. This is necessary in order to increase the amount of light received, to average the amplitude scintillations, and to decrease the signal fluctuations due to steering and breathing of the beam resulting from themal refraction.

The optical pulses thus received are detected by the photodiode (or a fast photomultiplier) (19) and displayed on a Tektronix Type 1S2 Sampling Plug-In. Since the maximum rate of fluctuation in the index of refraction is approximately $1 \mathrm{kHz}$ (3), the conditions of the atmosphere cannot change during one millisecond. Therefore, the atmospheric conditions can be considered "frozen" during the one millisecond sampling period and the high frequency pulses may be reconstructed at a lower frequency which can be handled by conventional circuitry without any loss of definition. The reconstructed pulse, taken from the 
vertical output of the sampler, is then fed into the distortion analyzer, which includes an automatic gain control to smooth the slower amplitude changes, and a distortion comparator to detect time smearing •

\section{DISTORTION ANALYZER DESIGN}

The Distortion Analyzer Module consists of three submodules, as shown in Figure 25, which are: (1) a transistor switch to eliminate transients during off time; (2) an automatic gain control (AGC) to smooth amplitude fluctuations; and (3) a comparator unit to compare the signal pulses to recorded reference pulses, with a threshold alarm to detect the difference (i.e., the distortion). The transistor switch is an emitter-follower with a supply which is controlled by the system logic. Thus, a signal is passed only when the logic is on, and when the logic is off, the output is grounded.

The AGC is a state-of-the-art analog multiplier (20) used in a divider configuration. Its inputs are the sampler output (from the transistor switch) and a voltage representing the normalizing or instantaneous optical intensity value. The more conventional choice of "limiting" or "feedback" AGC were not used because of the requirement of avoiding any possible distortions which could be caused by the AGC. The high frequency photodiode pulses were fed directly into the sampler with the best possible match; therefore any AGC used had to be incorporated after the low frequency output of the sampler, which consists of reconstructed pulses with a one millisecond duration and a repetition rate of $300 \mathrm{~Hz}$. Since the desirable AGC bandwidth is 


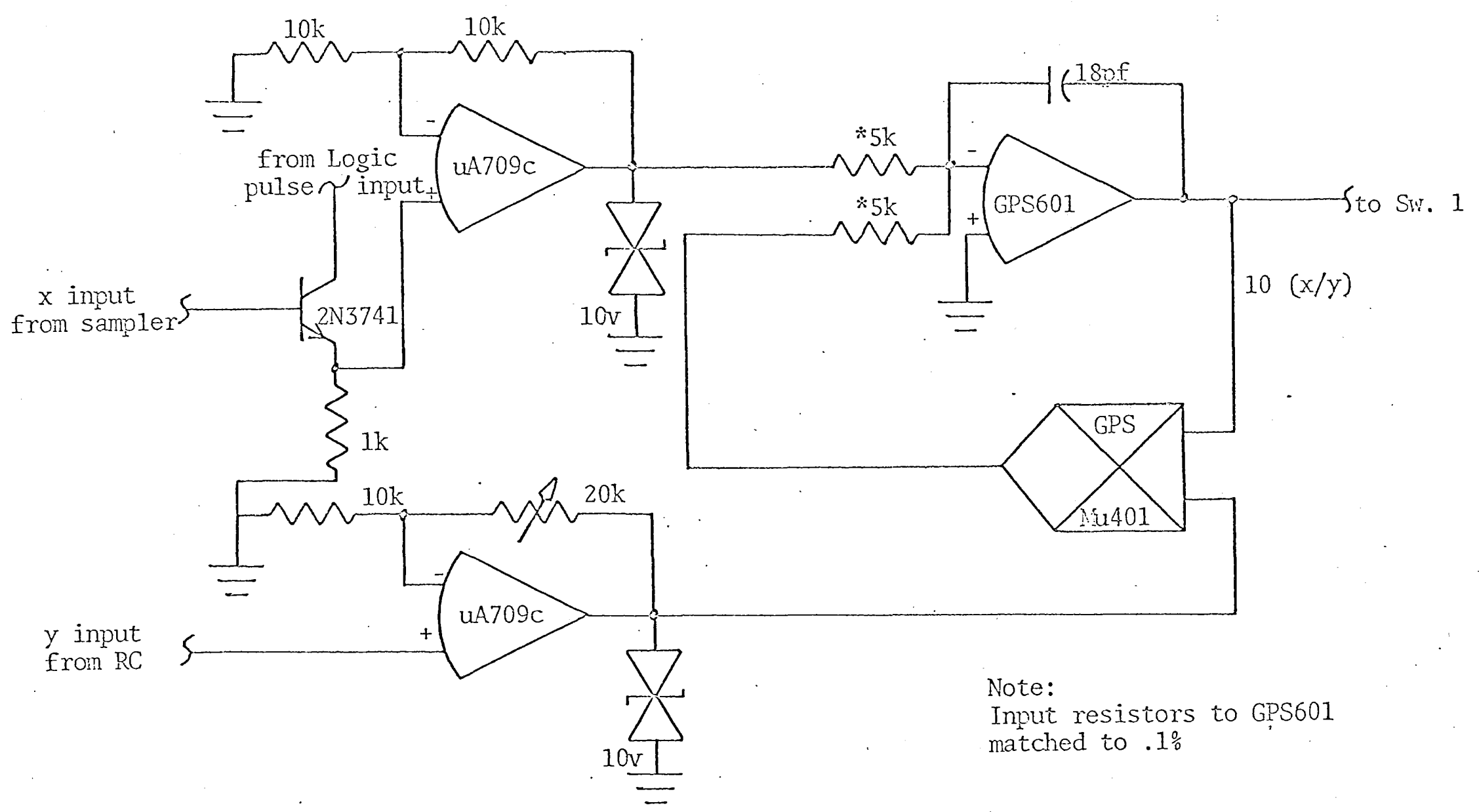

Figure 27. Automatic Gain Control - divider with transistor switch 
$1 \mathrm{kHz}$, corresponding to a frozen atmosphere, the reconstructed pulses cannot themselves be used for obtaining AGC information.

The solution used is to monitor the average photodetector current using the R-C low-pass filter shown in Figure 25. This becomes the denominator-input to the divider. The resistor and capacitor are chosen to give about a $10 \mathrm{kHz}$ break frequency with a +3 volt output leve1. This gives the divider a signal with a bandwidth greater than the maximum fluctuation rate, while leaving the $75 \mathrm{MHz}$ pulses unaffected by the R-C combination. If the photodiode is operated within its linear region, the instantaneous signal voltage is proportional to the average signal current, and therefore the ratio between signal voltage and average current will be a constant. The output of the AGC divider is this ratio of instantaneous signal to the average signal, which results in a constant-area pulse. Thus, by using this feedforward divider configuration, we have an AGC composed of conventional electronics which is capable of smoothing $1 \mathrm{kHz}$ fluctuations in a $300 \mathrm{~Hz}$ signal without inducing distortions. It does, however, require linearity of the photoreceiver.

The comparator consists of a subtractor, rectifier, integrator, and threshold detector (Figures 28, 29, 30, 31 and 32). The subtractor takes the arithmetic difference between the reference and AGC pulses, and also has a nominal-gain-control for equalization. This difference signal is fed into a highly linear full-wave analog rectifier (21), which gives the absolute value of the difference. This in turn is. fed into a resetable integrator which is time-scaled by 1000 to give reasonable gain during the short integration time (i.e., 


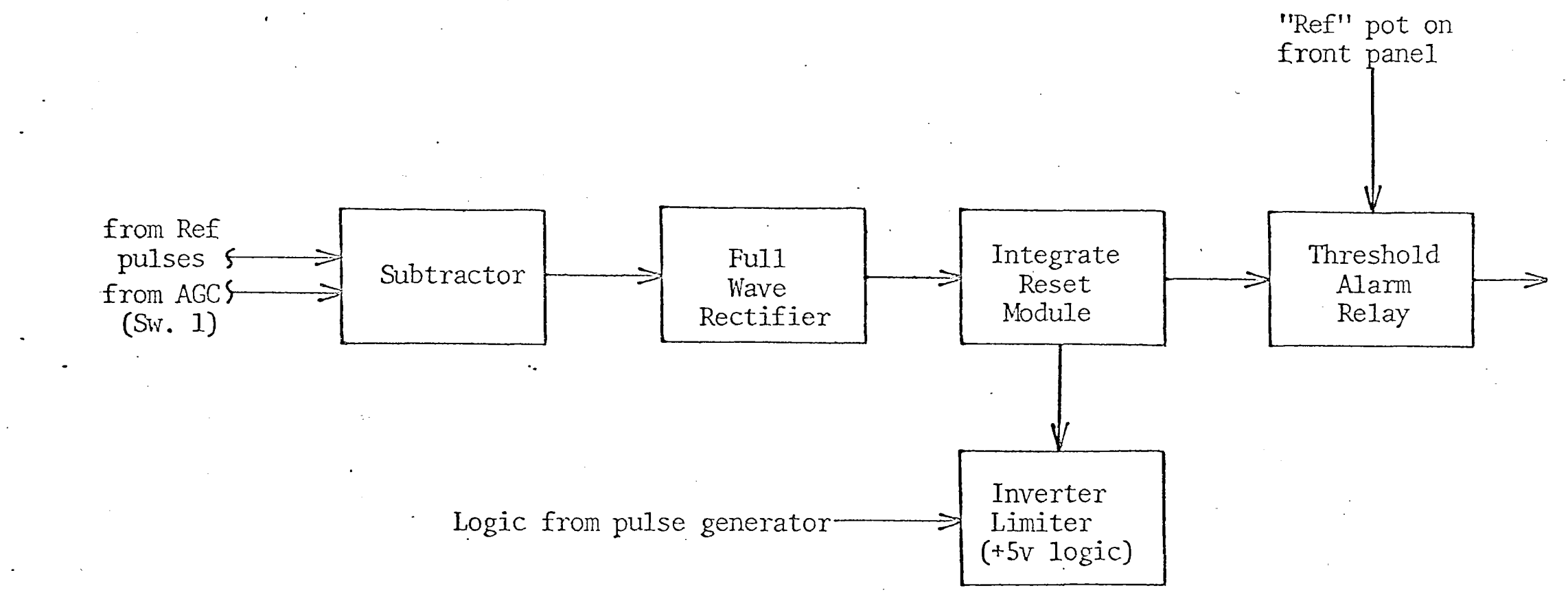

Figure 28. Block diagram of Short Pulse System Comparator 


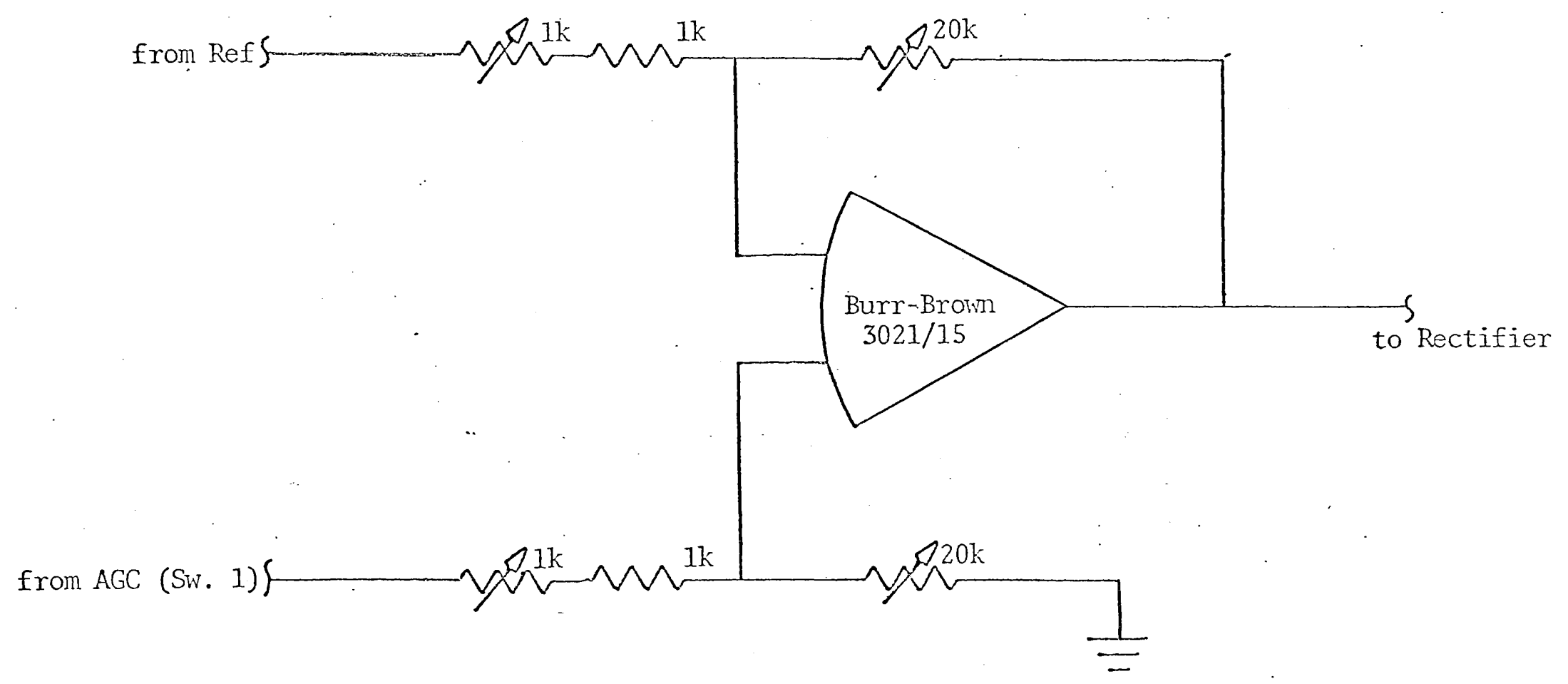

Figure 29. Short Pulse System Subtractor 


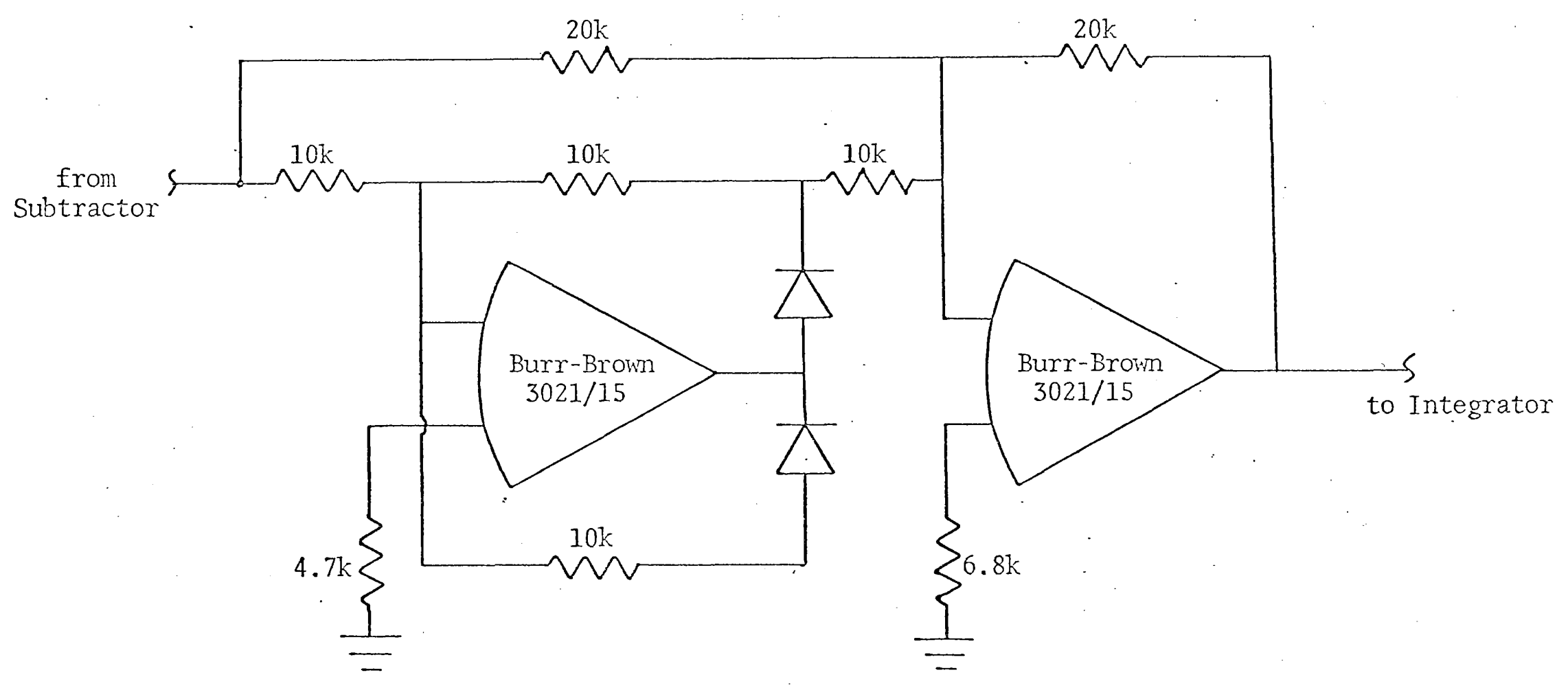

Figure 30. Short Pulse System Fullwave Rectifier 


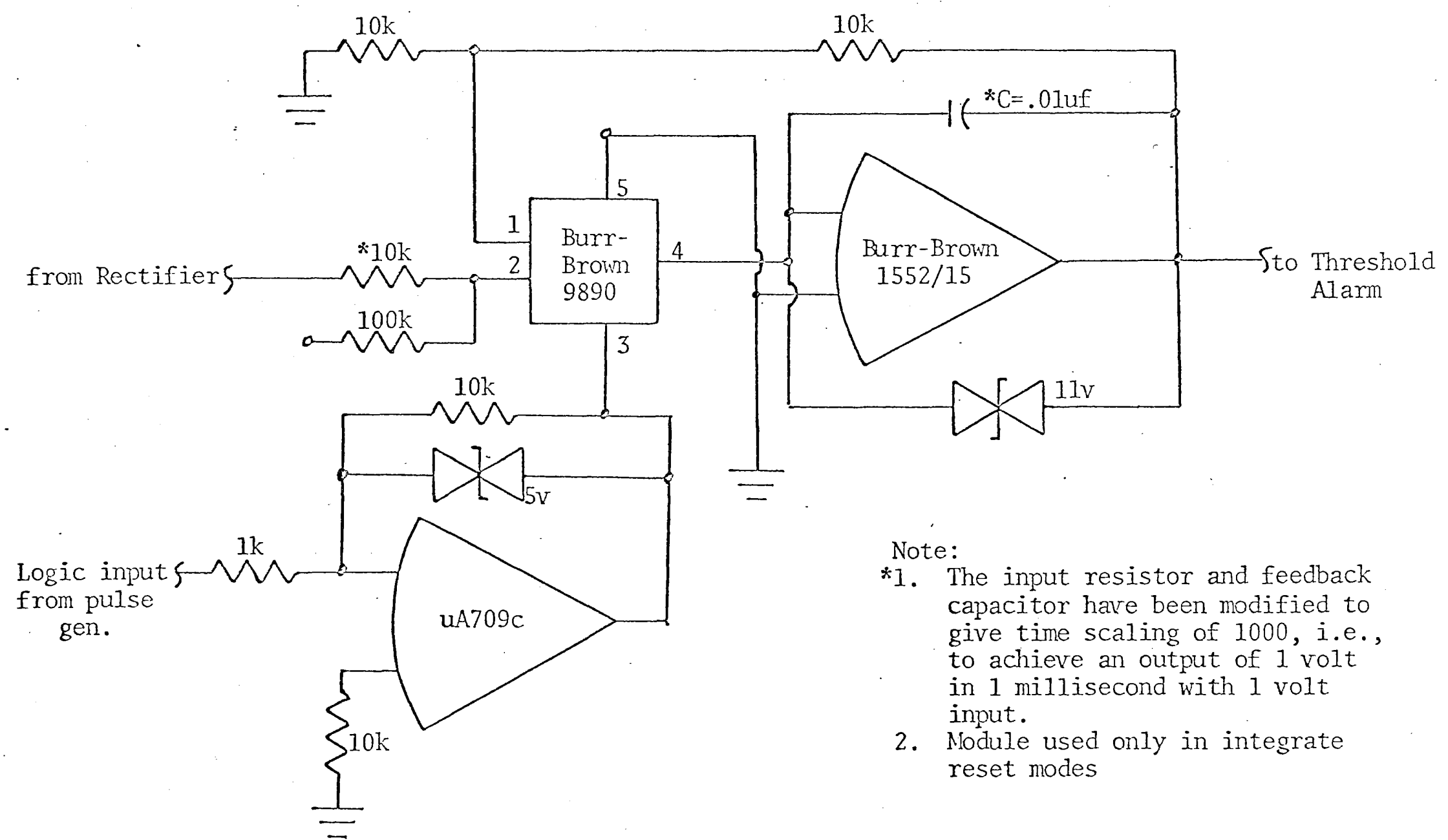

Figure 31. Short Pulse System Resetable Integrator 


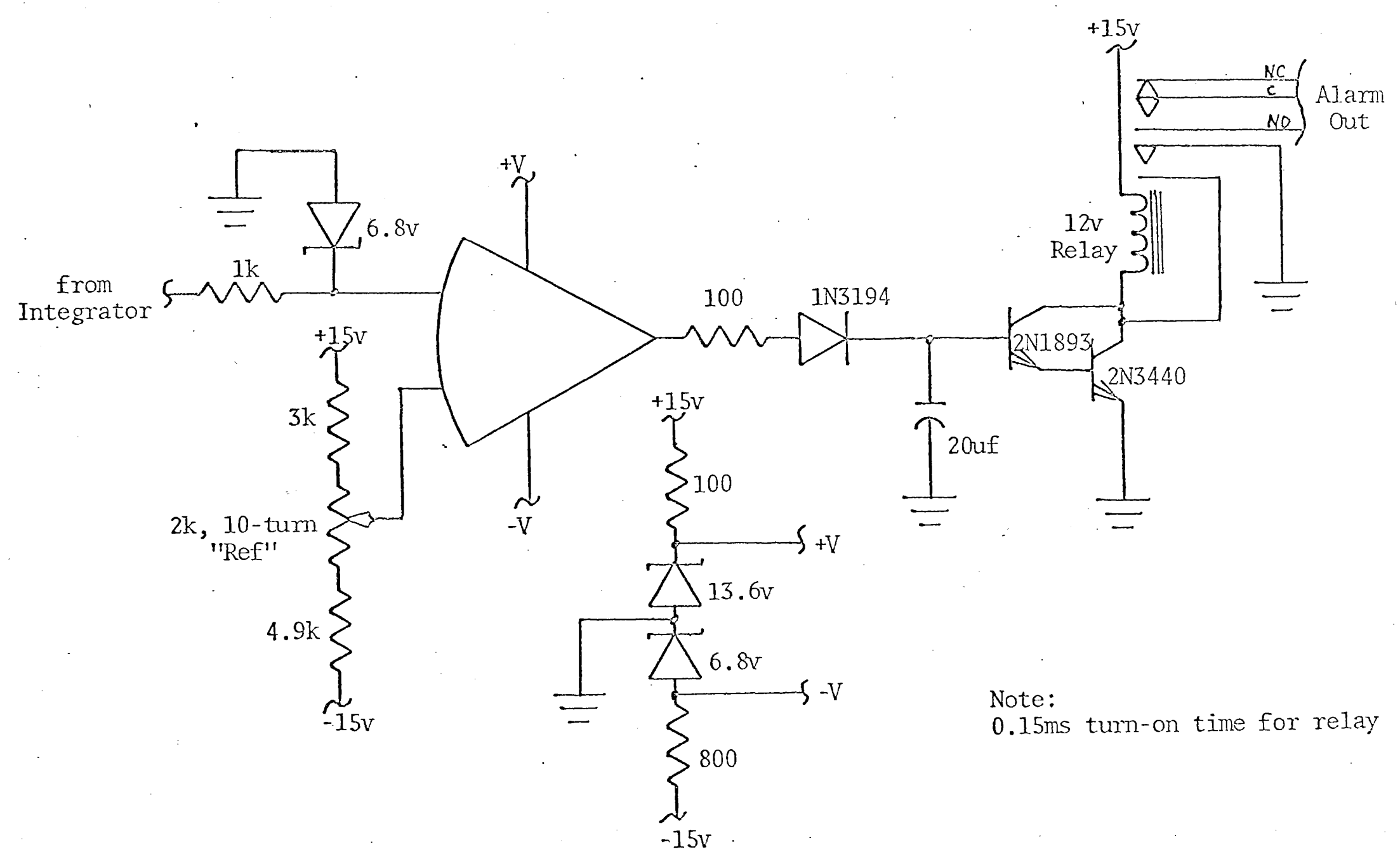

Figure 32. Short Pulse System Threshold Alarm 
one volt output in one millisecond with a one volt input), and which is reset after every reconstructed signal pulse, so that each new pulse is examined individually. The integrator output is finally fed into a high-speed, variable-threshold comparator which in turn actuates a relay if the threshold is exceeded. This relay is used to alert the operator (or mark tape) so that the anomaly can be studied. Also, the output from the integrator can be metered to give average differences, etc. The resetable integrator is controlled by a pulse logic system. It is held in the reset mode until the logic switches to +5 volts, then integrates while the logic stays at +5 volts, and resets when the logic drops to zero. This method eliminates interference from events (switching transients, etc.) which are outside the "time-window" (i.e., pulse period) we wish to observe.

\section{RECEIVER OPERATION}

The detection of pulse-distortion is a two-step process. First, ideal or reference pulses are recorded in the laboratory, and then these pulses are compared to the received pulses after transmission through the atmosphere. The pulses will be recorded on a FM tape recorder to provide baseband response and eliminate distortion due to the recorder. (Our test runs have used an AC-coupled recorder with compensation to equalize the sag.) The transmitted pulses are recorded in phase with the reference pulse so that anomalies can be investigated and a permanent record kept. The actual seven-mile experiment will begin on Sylvania's long path in the near future. 
The logic pulses (Figure 33) are used to determine the timewindow (i.e., the period of comparison) and sweep rate (the time between windows). This logic is generated by a Tektronix Type 114 audio pulse generator. In the laboratory or reference recording, the 114 is allowed to free-run at a rate slightly slower than the normal repetition rate of the sampler, which allows all systems to settle down before repeating the trigger. Therefore, the time stability of the recordings is as good as that of the pulse generator.

The taking of a complete pulse sample is initiated by the leading edge of the logic pulse, which requires a slight modification of the "single-sweep" mode of the sampler and thus its repetition rate and start time are controlled by this logic pulse generator. Also, since the transistor switch and resetable integrator are controlled by the logic pulse, it becomes a "time window." By altering the pulse width control on the 114 and by adjusting the time scale and position controls of the 1S2 sampler, one can electronically compare the entire reconstructed (sampled) pulses or any part of those pulses.

In the field, the 114 is triggered by the reference pulse from tape recorder No. I, which thus initiates the sampling of the transmitted pulse in phase with the reference pulse as shown in Figure 33 The 114 has an external trigger input, but does not contain an internally adjustable trigger. Therefore, trigger pulses of proper shape must be externally provided. This can be accomplished in two ways: (1) through the use of an extemal oscilloscope which is triggered by the reference pulses and which also generates a trigger pulse output (e.g., a Tektronix Type 556 oscilloscope), or (2) through 


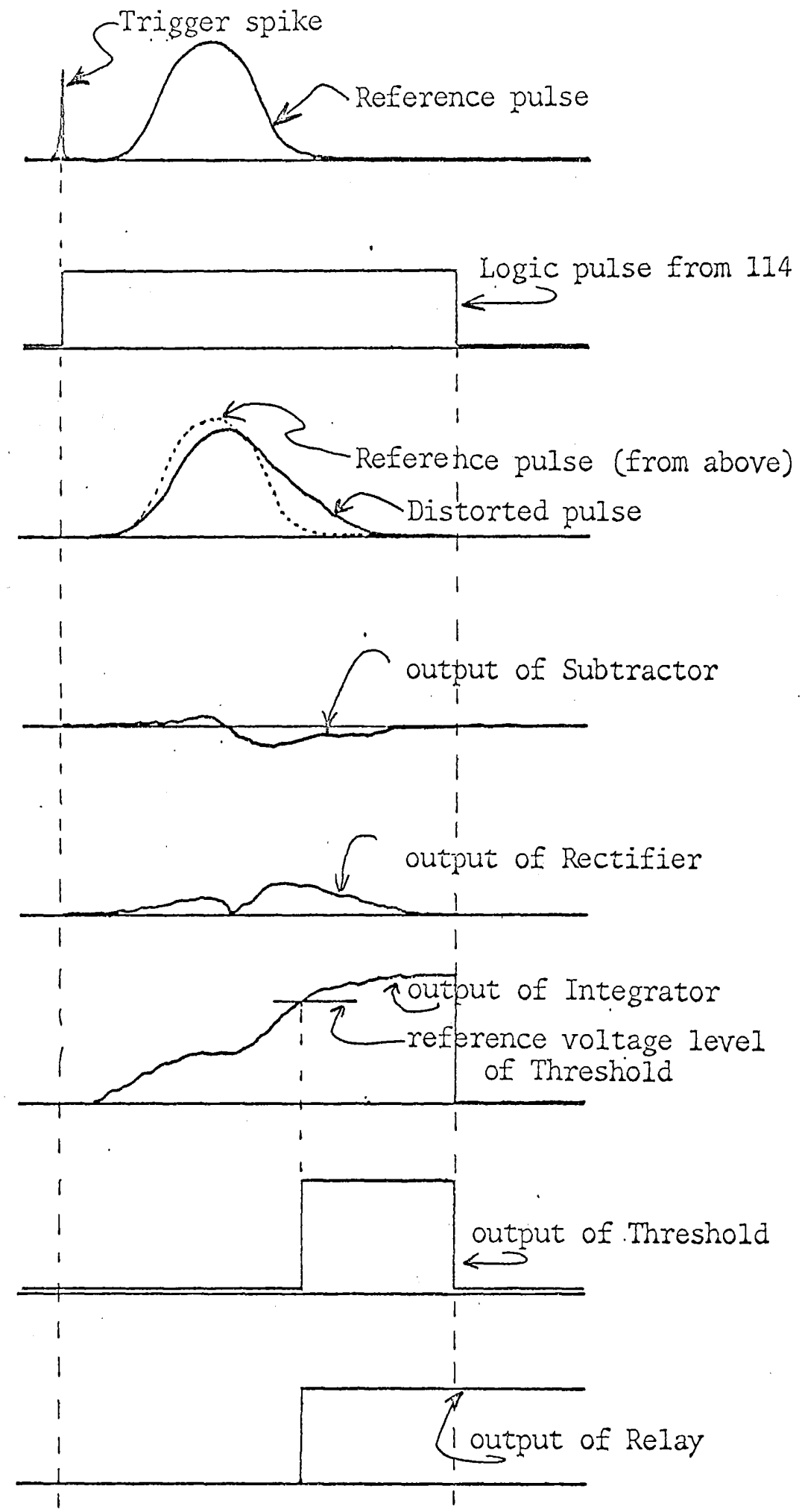

Figure 33. Short Pulse Systèm logic waveforms 
the use of a recorded pulse on the reference recording or an adjacent channel which is of appropriate amplitude and risetime to trigger the 114 directly.

Either method provides a trigger pulse near the start of the reference pulse; however, the recorded pulse is preferable since it can then slightly preceed the reference pulse thus simplifying the system operation. This trigger pulse can be taken directly from the 114 during the recording of the reference tape since the 114 generates a trigger pulse output on the leading edge of the logic pulse. Thus the reference and transmitted pulse reconstructions will start in phase and by adjusting the horizontal position control on the sampler, the signal pulse reconstruction can be moved in time to coincide with the reference pulse itself. Therefore, it is not necessary to use a delay line to obtain phase-match between the two pulses. The pulses can be compared "live" at the receiver site and/or recorded for later comparison. It is also necessary to re-record all reference data during the transmitted pulse recording process since there is a time lag between read and record tape heads.

The comparator section of the distortion analyzer is designed such that it may be used following the AGC for "live" comparison or independent of AGC for comparison of pre-recorded data. Thus the AGC and comparator are separate units and may be calibrated independently. Calibration of the AGC using square waves has shown approximately a two per-cent change with a ninety per-cent reduction of signal. Calibration using the optical setup and neutral density filters has shown approximately a five per-cent change with a fifty per-cent 
reduction of signal; however, we were unable to judge this accurately due to the five to seven per-cent instability of the mode-locked laser output. Also, this effect is enhanced by a ten per-cent non-linearity in the photodiode. For the actual experiment, a wideband crossed-field photomultiplier will be used and should not have this non-linearity (19). Also, the laser will be quieted using improved mode-locking techniques.

Calibration of the comparator using five-volt square waves has shown a repeatable sensitivity to less than one-half-per-cent change in area. Further calibration checks show the signal out of the integrator to be proportional to the change in area, and since the threshold reference potentiometer is a linear function of its setting, that potentiometer can be calibrated directly in terms of differential area. This would be true of any type of wave form; however, gaussian pulse distortions differ from the square wave differential area by a numerical factor. Therefore, the actual calibration of the comparator should be completed with known distortion of a gaussian wave form.

The most direct method of accomplishing this is to compare, in the laboratory, the taped reference to a pulse which is distorted by transmission-line mismatch (i.e., the distortion of the two nanosecond pulses themselves). A second and possibly simpler method of distorting the pulses by a known amount is to split ten per-cent of the light, use mirrors to delay it by a known path-difference, and then to recombine it with the main beam and detect the result with the photodiode. This would approximate single scattering with a variable path difference. This has not been done because we do not yet have the FM recorder 
and conventional "high fidelity" recorders, being ac coupled, induce too much distortion of their own to permit this calibration. Another factor in the requirement of a FM tape recorder is that flutter, wow, and other tape speed fluctuations, common to conventional 'high fidelity" tape recorders, appear exactly the same as pulse distortions and cannot be allowed. It is thus necessary that this calibration be completed at Sylvania.

In summary, a system capable of detecting pulse-area differences of less than one per-cent, and with order-of-magnitude, distortionfree smoothing of signal fluctuations, has been demonstrated. The interfacing of this unit with the final recording electronics has proceeded smoothly, and ultimate system performance should be limited only by the instability of the laser transmitter.

The system is immediately adaptable to short optical pulses and faster photodetectors and samplers, and may eventually be used with mode-locked YAG lasers (22) to detect anomalies in 30 picosecond pulses. This would correspond to the detection of time-dispersions on the order of $10^{-11}$ seconds, depending upon detector bandwidth. 


\section{A SELECTED BIBLIOGRAPHY}

(1) H. Hodara, "Laser Wave Propagation," Proc. IEEE, vo1. 54, March 1966, pp. 368-375.

(2) P. Beckmann, "Signal Degeneration in Laser Beams Propagated Through a Turbulent Atmosphere," J. Res. NBS (Radio Science), USNC-URSI, vol. 69, pt. D, ApriI $196 \overline{5}$.

(3) V. I. Tatarski, Wave Propagation in Turbulent Medium. New York: McGraw-Hill Book Co., Inc., 1961.

(4) L. A. Chernov, Wave Propagation in a Random Medium. New York: McGraw-Hil1 Book Co., Inc., 1960.

(5) D. A. DeWolf, "Saturation of Irradiance Fluctuations Due to Turbulent Atmosphere," J. Opt. Soc. Am., vol. 58, April 1968, p. 461.

(6) W. P. Brown, "Validity of the Rytov Approximation," J. Opt. Soc. Am., vol. 57, December 1967, p. 1477.

(7) MIT/NASA Workshop on Optical Communications, August 5-16, 1968, Williamstown, Mass. (Proceedings to be Published).

(8) E. Hoversten, Private Communication, Electrical Engineering Dept., MIT.

(9) A. Holland, Private Communication, NASA/ERC.

(10) R. R. Carter, et al., Ultrafast Pulsed Laser System, Sylvania Electronic Systems, Inc., Mt. View, California, May 1968.

(11) R. S. Lawrence, Private Communication, ESSA.

(12) D. L. Fried, "Propagation of an Infinite Plane Wave in a Randomly Inhomogeneous Medium," J. Opt. Soc. Am., vol. 56, December 1966, p. 1667.

(13) D. L. Fried, Private Communication, Autonetics.

(14) R. A. Schmeltzer, 'Means, Variances, and Covariances for Laser Beam Propagation through a Random Medium," Quarterly of Applied Mathematics, vol. XXIV, January 1967, p. 339 . 
(15) D. L. Fried, et al., 'Measurements of Laser Beam Scintillation in the Atmosphere," J. Opt. Soc. Am., vol. 57, June 1967, p. 787

(16) S. E. Harris, "Stabilization and Modulation of Laser Oscillators by Intermal Time-Varying Perturbation," Proc. IEEE, vol. 54, October 1966, p. 1401.

(17) H. Kogelnik and T. Li, "Laser Beams and Resonators," Proc. IEEE, vol. 54, October 1966, p. 1312.

(18) A. L: Bloom, "Gas Lasers," Proc. IEEE, vo1. 54, October 1966, p. 1262 .

(19) J. R. Kerr, 'Microwave-Bandwidth Optical Receiver Systems," Proc. IEEE, vol. 55, October 1967, p. 1686.

(20) Burr-Brown March 1968 Catalog. Tucson, Arizona: Burr-Brown Research Corporation, 1968.

(21) Handbook of Operational Amplifier Applications. Tucson, Arizona: Burr-Brown Research Corporation, 1963.

(22) Z. J. Kiss and R. J. Pressley, "Crystalline Solid Lasers," Proc. IEEE, vol. 54, October 1966, p. 1236. 